\title{
Infralimbic prefrontal cortex structural and functional connectivity with the limbic forebrain: a combined viral genetic and optogenetic analysis
}

\author{
Miranda Wood ${ }^{1}$, Othman Adil ${ }^{1}$, Tyler Wallace ${ }^{2}$, Sarah Fourman $^{1}$, Steven P. Wilson ${ }^{3}$, James \\ P. Herman ${ }^{1}$, and Brent Myers ${ }^{2}$ \\ ${ }^{1}$ Psychiatry and Behavioral Neuroscience, University of Cincinnati, Cincinnati, $\mathrm{OH}$, USA \\ ${ }^{2}$ Biomedical Sciences, Colorado State University, Fort Collins, CO, USA \\ ${ }^{3}$ Pharmacology, Physiology, and Neuroscience, University of South Carolina, Columbia, SC, USA
}

\section{Abstract}

The medial prefrontal cortex is critical for contextual appraisal, executive function, and goaldirected behavior. Additionally, the infralimbic (IL) subregion of the prefrontal cortex has been implicated in stress responding, mood, and fear memory. However, the specific circuit mechanisms that mediate these effects are largely unknown. To date, IL output to the limbic forebrain has been examined largely qualitatively or within a restricted number of sites. To quantify IL presynaptic input to structures throughout the forebrain, we utilized a lentiviral construct expressing synaptophysin-mCherry. Thus, allowing quantification of IL efferents that are specifically synaptic, as opposed to fibers of passage. Additionally, this approach permitted the determination of IL innervation on a sub-structural level within the multiple heterogeneous limbic nuclei. To examine the functional output of the IL, optogenetic activation of IL projections was followed by quantification of neuronal activation throughout the limbic forebrain via fos-related antigen (Fra). Quantification of synaptophysin-mCherry indicated that the IL provides robust synaptic input to a number of regions within the thalamus, hypothalamus, amygdala, and bed nucleus of the stria terminalis, with limited input to the hippocampus and nucleus accumbens. Furthermore, there was high concordance between structural connectivity and functional activation. Interestingly, some regions receiving substantial synaptic input did not exhibit significant increases in Fra immunoreactivity. Collectively, these studies represent a step toward a comprehensive and quantitative analysis of output circuits. This large scale efferent quantification or 'projectome' also opens the door for data-driven analyses of the downstream synaptic mechanisms that mediate the integrative aspects of cortico-limbic interactions.

\footnotetext{
Address for correspondence Brent Myers, Ph.D., Department of Biomedical Sciences, Colorado State University, 1617 Campus Delivery, Fort Collins, CO 80523, brent.myers@colostate.edu.

Conflict of interest: The authors have no conflicts of interest to declare.

Ethical approval: All animal procedures and protocols were approved by the Institutional Animal Care and Use Committee and comply with the National Institutes of Health Guidelines for the Care and Use of Laboratory Animals
} 


\section{Keywords}

anterograde; fos-related antigen; rat; synaptophysin

\section{Introduction}

The medial prefrontal cortex (mPFC) coordinates executive processes based on contextual stimuli to generate goal-directed behavior (Damasio et al. 1996; Wood and Grafman 2003). In addition to cognition and behavioral control, brain imaging studies have implicated mPFC activity in the pathophysiology of mood and anxiety disorders, including post-traumatic stress disorder and major depressive disorder (MDD). Specifically, activity of the subgenual cingulate region (Brodmann area 25 [BA25]) of mPFC is implicated in negative affect and treatment-resistant MDD (Mayberg et al. 1999, 2005). Further, BA25 exhibits decreased activity, volume, and expression of immediate-early genes in MDD (Drevets et al. 1997, 2008; Covington et al. 2010). Intriguingly, deep brain stimulation of BA25 in patients with treatment-resistant MDD reduces depressive symptoms (Mayberg et al. 2005). Collectively, these studies indicate that the structure and activity of mPFC subregions, particularly BA25, are critical for behavioral regulation and associated affective pathology.

Based on comparative analysis, the rodent mPFC is divided into subregions with proposed homologies to Brodmann areas. For instance, infralimbic cortex (IL) is phylogenetically similar to BA25 in terms of agranular structure and thalamic connectivity (Uylings et al. 2003; Vertes 2004; Gabbott et al. 2005). Given these similarities, rodent studies have begun to investigate the role of the IL subregion in behaviors related to depression and emotional memory (Hamani et al. 2010; Sotres-Bayon and Quirk 2010; McKlveen et al. 2013). Importantly, these effects appear to be selective to the IL over neighboring limbic cortical regions, such as prelimbic cortex (PL), and are highly-dependent on specific projections (Laurent and Westbrook 2009; McKlveen et al. 2013; Fuchikami et al. 2015). For instance, evoked $\mathrm{mPFC}$ glutamate release at prefrontal synapses in the dorsal raphe promotes active coping while stimulation of prefrontal projections to the lateral habenula (LHab) increases passive behavior (Warden et al. 2012). Moreover, the IL is necessary for the extinction of associative fear, a process proposed to depend on differential innervation of amygdala regions involved in emotional memory (Sierra-Mercado et al. 2011). Overall, there is substantial and growing evidence for subregional specialization within the mPFC that indicates the IL is an important regulator of behavioral adaptation (Myers et al. 2014b; McKlveen et al. 2015).

Traditional tracing studies have identified IL projections to major limbic behavioral- and homeostatic-regulatory regions such as the amygdala, bed nucleus of the stria terminalis (BST), and hypothalamic nuclei (Hurley et al. 1991; Vertes 2004; Myers et al. 2014a). The methods used to examine structural output have included either multisynaptic tracers such as rabies virus (Dum et al. 2016) that spread transsynaptically, or monosynaptic anterograde tracers such as the plant lectin Phaseolus vulgaris leucoagglutinin (Hurley et al. 1991; Vertes 2004). Although monosynaptic anterograde tracers label axonal processes and presynaptic terminals, distinguishing between fibers of passage and synapses can be subjective. As a 
result of the non-specific subcellular targeting of lectins within neuronal projections and the qualitative nature of examining tracer immunoreactivity, the quantification of efferent output has been elusive. In the current study, a viral genetic approach is used to drive the expression of mCherry conjugated to synaptophysin, a glycoprotein present in the vesicular membranes of presynaptic terminals (Wiedenmann and Franke 1985; Carvalho-Netto et al. 2011). This genetically-encoded reporter of presynaptic input is used to quantify the relative weight of projections to the many subnuclei of limbic forebrain regions, with a focus on subcortical nuclei implicated in behavioral and homeostatic processes. Additionally, optogenetic stimulation of IL glutamatergic projection neurons is employed to assess cellular activation throughout the limbic forebrain. Quantification of fos-related antigen (Fra) protein expression is used to map functional connectivity, as well as compare structure-function relationships.

\section{Materials and methods}

\section{Animals}

Male Sprague-Dawley rats were obtained from Harlan (Indianapolis, IN) with weights ranging from 350-400 g. Rats were housed individually in shoebox cages in a temperatureand humidity-controlled room with a 12 hour light-dark cycle (lights on at 0600h, off at $1800 \mathrm{~h}$ ) and food and water ad libitum. All procedures and protocols were approved by University of Cincinnati Institutional Animal Care and Use Committee and comply with the National Institutes of Health Guidelines for the Care and Use of Laboratory Animals.

\section{Experiment 1: lentivirus validation}

Lentiviral construct-A lentivirus transfer vector, based on a third-generation selfinactivating transfer vector (de Almeida et al. 2001), was designed to over-express a synaptophysin-mCherry fusion protein. The vector uses the phosphoglycerate kinase-1 promoter which expresses well in rat brain and is primarily neuronal (Krause et al. 2011; Grillo et al. 2015; Myers et al. 2017). Both rat synaptophysin (Open Biosystems MRN176899237971, clone 7936715) and mCherry (Clontech pmCherry-1) cDNAs were amplified by PCR, using primers designed to substitute GTG for the stop codon of the synaptophysin cDNA immediately upstream of the mCherry ATG. The two PCR fragments were cloned into the lentivirus transfer vector using Clontech Advantage HD Polymerase (Mountain View, CA). Viruses were generated by transfection of the transfer vector with three packaging plasmids (Addgene, Cambridge, MA), psPAX2, pRSV-Rev and pMD2.G, into 293 T cells. Viruses were concentrated by high-speed centrifugation, purified by further centrifugation through 20\% sucrose/Dulbecco's phosphate buffered saline (DPBS) and stored in $10 \%$ sucrose/DPBS at $-80^{\circ}$. Virus particle concentrations were determined by quantitative real-time PCR for proviral DNA $24 \mathrm{~h}$ following transduction of $293 \mathrm{~T}$ cells and are expressed as transducing units per microliter $(\mathrm{tu} / \mu \mathrm{l})$.

Stereotaxic surgery—Rats were anesthetized with ketamine $(90 \mathrm{mg} / \mathrm{kg}$, i.p.) and xylazine (10 mg/kg, i.p.), followed by administration of analgesia (butorphanol, s.c.) and antibiotic (gentamicin, i.m.). Microinjections of lentivirus expressing synaptophysinmCherry fusion protein $\left(1 \mu \mathrm{L}, 5.2 \times 10^{6} \mathrm{tu} / \mu \mathrm{l}\right.$ titer $)$ were targeted to the IL $(+2.8 \mathrm{~mm}$ from 
bregma, $0.6 \mathrm{~mm}$ from midline, and $-4.2 \mathrm{~mm}$ from dura) with a 25 -gauge, $2-\mu \mathrm{L}$ flat tip Hamilton syringe (Reno, NV). After the needle was in place for $5 \mathrm{~min}$, virus was infused with a microinjection unit (Model 5001; Kopf, Tujunga, CA) at a rate of $5 \mathrm{~min} / \mu \mathrm{L}$. After each injection, the needle was left in place for an additional 5 min to allow for viral diffusion. Rats were given six weeks of recovery, corresponding to timeframes previously used for similar lentiviral systems (Krause et al. 2011; Myers et al. 2017).

Tissue collection-Rats were given an overdose of sodium pentobarbital and perfused transcardially with $0.9 \%$ saline followed by $4.0 \%$ paraformaldehyde in $0.1 \mathrm{M}$ PBS. After brains were removed, they were post-fixed in $4.0 \%$ paraformaldehyde for 24 hours at room temperature, followed by storage in $30 \%$ sucrose in PBS at $4^{\circ} \mathrm{C}$. Coronal sections were made on a freezing microtome at $30 \mu \mathrm{m}$ thickness and then stored in cryoprotectant solution at $-20^{\circ} \mathrm{C}$ until processing.

Immunohistochemistry-To examine viral expression pattern at injection and terminal sites, single immunolabeling of neuronal nuclear protein (NeuN) was carried out. Tissue sections were rinsed in $50 \mathrm{mM}$ potassium phosphate buffered saline (KPBS) and incubated in blocking buffer (50 mM KPBS, 0.1\% BSA, and 0.2\% TritonX-100) for $1 \mathrm{~h}$ at room temperature. Sections were then placed in mouse monoclonal anti-NeuN antibody (1:200; Millipore, Billerica, MA) overnight at $4^{\circ} \mathrm{C}$. Following incubation, sections were rinsed and placed into Cy5-conjugated goat anti-mouse IgG (1:500; Jackson ImmunoResearch, West Grove, PA) for $30 \mathrm{~min}$. Sections were then rinsed, mounted onto slides, and cover slipped. In order to compare synaptophysin-mCherry expression with synaptophysin immunolabeling, immunohistochemistry for synaptophysin was carried out in a similar manner as above except with rabbit monoclonal anti-synaptophysin (1:200; Abcam, Cambridge, MA) followed by Alexa488-conjugated goat anti-rabbit IgG (1:500; Jackson ImmunoResearch) and DAPI (1 $\mu \mathrm{g} / \mathrm{ml}$ for $10 \mathrm{~min}$; Millipore, Billerica, MA).

Microscopy-Synaptophysin-mCherry, antibody-labeled synaptophysin, and DAPI were imaged in the medialdorsal thalamus (MD) on a Zeiss Axio Imager Z2 microscope. Highmagnification optical sections were captured in the posterior hypothalamus $(\mathrm{PH})$ to localize synaptophysin-mCherry and synaptophysin immunofluorescence within a single optical section ( $0.5 \mu \mathrm{m}$ thickness). Additionally, tiled images were obtained of synaptophysinmCherry expression in the amygdala. Photomicrographs of NeuN and synaptophysinmCherry, as well as viral injection sites were imaged with a Zeiss Axio Observer microscope. Brightness and contrast of representative images were enhanced uniformly with Adobe Photoshop (CC 14.2).

\section{Experiment 2: structural connectivity}

Following validation of the viral tracing approach in experiment 1 , cases that exhibited successful viral transfection limited to the boundaries of the IL ( $n=3$ rats) were selected for quantitative analysis. Importantly, cases with viral spread into the dorsal portions of mPFC were removed prior to analysis. One case each was chosen from rats that received either bilateral, unilateral left, or unilateral right injections. Structures were delineated based 
primarily on the atlas of Swanson (Swanson 2004) with supplementation from Paxinos and Watson (Paxinos and Watson 1998).

Microscopy—Digital images were captured with a Zeiss Axio Observer microscope using optical sectioning (40x objective) to determine the percent area occupied by synaptophysinmCherry in single z-planes ( $0.5 \mu \mathrm{m}$ thickness). Images were collected along the rostralcaudal gradient of each structure, typically representing a rostral, middle, and caudal portion of each nucleus. Based on the length of a given region, as few as 2 or as many as 5 image stacks were collected ipsilateral to each injection site.

Quantification-To quantify the regional density of presynaptic input, optical sectioning was used to collect z-axis image stacks with $0.5 \mu \mathrm{m}$ thick image planes. The middle 5 optical slices from each image stack were processed individually in ImageJ Fiji (ver $1.51 \mathrm{~N}$ ). The middle 5 optical sections were chosen based on a center-out focusing strategy as the most high-quality images collected across all cases. Each image was thresholded and the percent area occupied by fluorescence was quantified by a blinded observer. The 5 values were then averaged to yield a mean percent area for each location relative to bregma. For regions of interest, the mean percent area from each location along the rostral-caudal gradient was averaged to yield a regional percent area value (5 optical sections/z-stack, 2-5 z-stacks/structure) in each of the 3 cases. These values were then corrected for the case median percent area of all regions sampled to account for between-case variation in construct expression.

Data analysis-The regional corrected percent area of synaptophysin-mCherry expression was averaged across all 3 cases and this mean was rank plotted (ggplot2, version 2.2.1; Wickham 2009) followed by one-way analysis of variance (ANOVA) in R (version 3.3.2; Team 2014). Significant main effects of region were further analyzed by Bonferroni multiple comparison post-hoc with all regions compared to all other regions. Significance was matrix plotted to include $\mathrm{p}$ values from all comparisons $(a<0.05)$.

\section{Experiment 3: optogenetic efficacy and specificity}

Stereotaxic surgery-Surgical procedures were performed as described for experiment 1. Microinjections ( $2 \mu \mathrm{L}$ each) of adeno-associated virus (AAV) were targeted to the IL bilaterally ( $+2.8 \mathrm{~mm}$ from bregma, $+/-0.6 \mathrm{~mm}$ from midline, and $-4.2 \mathrm{~mm}$ from dura) with a 25-gauge, 2- $\mu \mathrm{L}$ flat tip syringe (Hamilton, Reno, NV) and microinjection unit (Model 5001; Kopf, Tujunga, CA). AAV5 constructs expressing YFP or channelrhodopsin-2 (ChR2) under the $\mathrm{Ca}^{2+} /$ calmodulin-dependent protein kinase II alpha (CaMKIIa) promoter were provided by the University of North Carolina Vector Core. Rats received either CAMKIIaChR2-YFP $(n=5)$ or CAMKIIa-YFP $(n=3)$. After each injection, the needle was left in place for $5 \mathrm{~min}$ to allow diffusion and then slowly withdrawn. Rats were then given six weeks of recovery before bilateral fiber-optic cannula implantation in the IL $(200 / 245 \mu \mathrm{m}$, $\mathrm{NA}=0.37,1.1 \mathrm{~mm}$ pitch, $4.5 \mathrm{~mm}$ protrusion, flat tip; Doric Lenses, Québec, Canada). Cannulas were secured with 2 metal screws and dental cement (Jet, Nashua, NH). Following one week of recovery, rats were handled daily for an additional week to acclimate to the stimulation procedure. 
Optogenetic protocol—Light pulses $(5 \mathrm{~ms}, 3 \mathrm{~mW}, 20 \mathrm{~Hz})$ were delivered through a branching fiber-optic patch cord ( $200 \mu \mathrm{m}$ core diameter, $\mathrm{NA}=0.37$, Doric Lenses) connected to a $473 \mathrm{~nm}$ LED driver (Doric Lenses) for $5 \mathrm{~min}$. Optic power was measured with a photodiode sensor (PM160, Thorlabs Inc, Newton, NJ) at cannula fiber tip, indicating light power exiting the cannula was approximately $2.2 \mathrm{~mW}$. Following stimulation, rats were given 90 min of recovery for optimal immediate-early gene protein expression and then euthanized with an overdose of sodium pentobarbital. Tissue collection was carried out as described for experiment 1 .

Immunohistochemistry-To assess activation of neurons expressing $\mathrm{ChR} 2$, immunofluorescent labeling of the intermediate-early gene c-Fos was carried out. Brain tissue was rinsed in $50 \mathrm{mM}$ KPBS (pH 7.4) and incubated in blocking solution (KPBS, $0.1 \%$ $\mathrm{BSA}$, and $0.2 \%$ TritonX-100) for $1 \mathrm{~h}$ at room temperature. Brain slices were then transferred into rabbit polyclonal anti-c-Fos (sc-52) primary antibody (1:1,000; Santa Cruz Biotechnology, Dallas, TX) overnight at $4^{\circ} \mathrm{C}$ (Jones et al. 2011). The next day, brain tissue was rinsed in KPBS and incubated in Cy3-conjugated donkey anti-rabbit $\operatorname{IgG}$ (1:500; Jackson ImmunoResearch) for $30 \mathrm{~min}$, followed by a final rinse. Sections were then mounted onto slides and cover slipped. To quantify specificity of the CaMKIIa promoter, immunofluorescent labeling of the GABAergic marker glutamic acid decarboxylase 67 (GAD67) was carried as described above using mouse monoclonal anti-GAD67 primary antibody (1:1,000; Millipore) and Cy3-conjugated donkey anti-mouse IgG (1:500; Jackson ImmunoResearch).

Microscopy-Fos and YFP imaging was carried out with a Zeiss Axio Imager Z2 microscope to verify optogenetic stimulation efficacy. To quantify specificity of the CaMKIIa promoter, optical sectioning ( $0.5 \mu \mathrm{m}$ thickness, $20 \mathrm{x}$ objective) was used to localize YFP and GAD67. For this measure, 10-section image stacks (5 $\mu \mathrm{m}$ total thickness) were collected bilaterally in the rostral, middle, and caudal portions of the IL from CaMKIIa-YFP-injected rats $(n=3)$.

Quantification-Examination of CaMKIIa promoter selectivity for projection neurons over interneurons was carried out by counting the total number of GAD67-positive neurons from each $5 \mu \mathrm{m}$ thick z-stack. These GABAergic cells were then examined for YFP fluorescence (6 images/animal from 3 animals) and the number of GAD67-positive and YFP/GAD67-colabeled cells was averaged.

\section{Experiment 4: functional connectivity}

After verifying optogenetic activation of IL projection neurons in experiment 3 , tissue from rats that received YFP-expressing $(n=3)$ or ChR2-expressing $(n=5)$ constructs was processed for quantitative analysis of functional connectivity.

Immunohistochemistry-Immediate-early gene immunoreactivity was used to regionally map neuronal activation as a function of IL stimulation. Briefly, sections were rinsed in KPBS, incubated in $0.03 \%$ hydrogen peroxide for $10 \mathrm{~min}$, rinsed, and placed in blocking solution (KPBS, $0.1 \%$ BSA, and $0.2 \%$ TritonX-100) for $1 \mathrm{~h}$ at room temperature. 
Tissue was then incubated in rabbit polyclonal anti-Fos (sc-253) antibody (1:1,000; Santa Cruz) overnight at $4^{\circ} \mathrm{C}$. This antibody labels multiple members of the Fra family, including Fra1, Fra2, c-Fos, and FosB (Ferrer et al. 1996; Budzikowski et al. 1998; Davern and Head 2007), and is a marker of recent neuronal activation (McClung et al. 2004). The following day, slices were rinsed and transferred into biotinylated goat anti-rabbit antibody (1:500; Vector Laboratories, Burlingame, $\mathrm{CA}$ ) for $1 \mathrm{~h}$ and then rinsed again. Slices were incubated in Vectastain ABC Solution (1:1,000; Vector Laboratories) for $1 \mathrm{~h}$ and then rinsed and incubated in diaminobenzidine and hydrogen peroxide ( $0.02 \%$ diaminobenzidine $/ 0.09 \%$ hydrogen peroxide in KPBS) for $10 \mathrm{~min}$. Following incubation, slices were rinsed, slide mounted, dehydrated in graded ethanol, and cover slipped.

Microscopy-To quantify Fra immunoreactivity, brightfield photomicrographs were captured with a Zeiss Axio Observer microscope (10x objective). For each region of interest, images were collected bilaterally in the rostral, middle, and caudal portions (6 images/ structure/animal). Regions with limited rostral-caudal distance were imaged in the rostral and caudal portions only. Representative images of Fra immunolabeling in limbic forebrain regions were scanned with a Zeiss Axio Imager Z2 microscope.

Quantification-To quantify the mean number of Fra-positive cells, regions of interest were outlined in digital images by an observer blinded to treatment. Following signal threshold determination, cell counts/area were quantified bilaterally through the rostral, middle, and caudal portions of each structure with Scion Image (Meyer Instruments, Houston, TX). These rostral-caudal samples were then averaged to provide the mean density of Fra-immunoreactive cells across all examined portions of each structure.

Data analysis-Fra-positive cell counts were analyzed using an unpaired t-test with differences between YFP and ChR2 treatment examined (Prism, Graphpad 5.0, La Jolla, $\mathrm{CA})$. The percent difference in regional cellular activation was calculated [(ChR2-YFP)/ YFP] and averaged across all $5 \mathrm{ChR} 2$-injected animals. This mean was rank plotted (ggplot2, version 2.2.1) followed by one-way ANOVA in R (version 3.3.2). Significant main effects of region were further analyzed by Bonferroni multiple comparison post-hoc with all regions compared to all other regions. Significance was matrix plotted to include $\mathrm{p}$ values from all comparisons with $a<0.05$.

\section{Results}

\section{Experiment 1: lentivirus validation}

Lentiviral construct-Following injection of lentiviral-packaged synaptophysin-mCherry into the IL, mCherry labeling was observed in the MD (Figure 1A) that colocalized with immunohistochemically-labeled synaptophysin (Figures 1B and 1C). Manders' overlap coefficient (MOC; Manders et al. 1992) value of 0.8883 reflected colocalization in terms of the proportion of the green signal coincident with a signal in the red channel over its total intensity. This colocalization analysis (Figure 1D) indicated a high degree of overlap between immunohistochemically-labeled synaptophysin and the vector-encoded synaptophysin-mCherry. A high-magnification optical section illustrates overlap of mCherry 
and Alexa488 signals (Figure 1E-G). Injection sites for the lentiviral-packaged synaptophysin-mCherry expression construct were visualized by the presence of mCherry in the IL (Figure 2A). As the reporter is trafficked to presynaptic terminals, mCherry fluorescence does not label the entire cell body of transfected neurons. In a region known to receive input from the IL, the PH (Myers et al. 2016), mCherry fluorescence labeled IL presynaptic terminals (Figure $2 \mathrm{~B}$ ). The ventromedial hypothalamus $(\mathrm{VMH})$ receives little mPFC input (Vertes 2004) and the area expressed almost no mCherry (Figure 2C). Construct expression was quantified throughout the limbic forebrain in single optical sections (Figure 2D) to determine inter- and intra-regional differences in IL presynaptic input.

\section{Experiment 2: structural connectivity}

Case 13-Injections for case 13 (Figure 3A and 3B) were matched with atlas maps modified from Swanson (Swanson 2004). The case was bilateral in the rostral $(+3.2) / \mathrm{mid}-$ rostral (+2.8) IL, primarily infecting cells in the deeper cortical layers (V/VI). The injections were confined to the IL without significant spread to neighboring regions. SynaptophysinmCherry was quantified bilaterally throughout the limbic forebrain, revealing distinct patterns of innervation (Figure 4). For each bregma level of each structure, 10 images were averaged to provide the relative synaptophysin-mCherry density (Mean \pm SEM). As expected, the neighboring PL received input from IL; however, the core and shell portions of the nucleus accumbens (NAc) were not targeted. The BST received input from the IL throughout its numerous subdivisions, including the anterior (anteromedial [am] and anterolateral [al]) and posterior nuclei (principal [pr], transverse [tr], and interfascicular [if]). The IL input to the hypothalamus was heterogeneous. There was mixed input at some levels of the anterior nuclei (e.g. preoptic) and almost no input to the paraventricular hypothalamus $(\mathrm{PVN})$ or VMH. Nuclei involved in pre-autonomic and behavioral functions such as the lateral hypothalamus ( $\mathrm{LH})$ and dorsomedial hypothalamus (DMH) were moderately targeted, with the densest innervation to the $\mathrm{PH}$. As thalamic connectivity is a defining feature of the mPFC (Uylings et al. 2003), the high level of innervation is fitting. Analysis was primarily focused on nuclei composing the limbic thalamus (Vertes et al. 2015) and dense pre-synaptic input was apparent particularly in the paraventricular thalamus (PVT) and MD, with most thalamic nuclei quantified exhibiting mCherry density above the case median. The LHab was included as a region of limbic epithalamus but projections to this region were modest. There was considerable variability within and amongst the nuclei of the amygdala. For instance, the basolateral amygdala (BLA) consistently received moderate input while the central amygdala $(\mathrm{CeA})$ was characterized by dense projections to the lateral $\mathrm{CeA}(\mathrm{CeL})$ and minimal input to the medial $\mathrm{CeA}(\mathrm{CeM})$. Interestingly, there was a considerable rostral-caudal gradient in the medial amygdala (MeA) whereby the anterior portions of the nucleus received sparse input and the more posterior portions exhibited dense mCherry expression. IL projections to the hippocampus were minimal with some observed in the dentate gyrus (DG).

Case 14-Case 14 received viral injection unilaterally in the left IL (Figure 5). The case was largely in the mid $(+2.8) /$ mid-caudal $(+2.2) \mathrm{IL}$, transfecting cells in the deep layers (V/ VI). The injection was confined to the IL without observable spread to other regions. Synaptophysin-mCherry was quantified throughout the left side of the limbic forebrain from 
5 images captured unilaterally for each bregma level of each structure. Analysis indicated similar yet distinct patterns of innervation compared to Case 13 (Figure 6). For instance, there was prominent input to the PL with minimal expression in NAc subregions. However, serial sampling in Case 14 produced more representation of BST subnuclei and dense synaptophysin-mCherry expression was measured in most regions. In particular, the ventral portion of the alBST and the dorsal portion of the amBST had high levels of input; in contrast, synaptophysin-mCherry expression was lower in the fusiform (fu) BST. The oval (ov) BST and posterior regions (if, tr, pr) also received substantial input. The hypothalamic projections of the IL were varied. The anterior hypothalamic nucleus (AHN), PVN, and VMH received very little input, while the medial preoptic area (mPOA), medial preoptic nucleus (MPN), LH, and DMH had expression levels above the median. As in case 13, Case 14 showed the densest hypothalamic projections targeting the PH. Innervation of the thalamus was present with most regions showing moderate/dense mCherry expression. The amygdala again displayed heterogeneous IL input both across and within nuclei. In case 14, the lateral amygdala (LA) and rostral basomedial amygdala (BMA) exhibited more robust innervation than case 13, while the selective targeting of the $\mathrm{CeL}$ and posterodorsal (pd) $\mathrm{MeA}$ was even more pronounced. Input to the hippocampus was consistently low across all regions.

Case 41-The injection for case 41 is shown in a representative photomicrograph (Figure 7). Case 41 received lentiviral injection into the right rostral (+3.2)/mid-rostral (+2.8) IL, primarily transfecting cells in the deep layers (V/VI). The injection was confined to the IL with minimal spread to neighboring regions. Synaptophysin-mCherry was quantified throughout the right limbic forebrain in 5 images captured unilaterally for each bregma level of each structure (Figure 8). As in previous cases, there was considerable input to PL and almost no innervation of the NAc. The BST exhibited IL input, primarily in the anterodorsal (ad) divisions, as well as fu and ov nuclei. Although IL projections to the hypothalamus were low in many nuclei, specific areas showed above-median input, including the mPOA and LH. Aligning with previous cases, the greatest hypothalamic projection of the IL was to the PH. Thalamic input from the IL was not as consistently robust as previous cases; however, many areas showed moderate input and the MD exhibited high synaptophysinmCherry expression. Projections to the amygdala were similar to previous cases, characterized by variable input throughout the BLA, LA, and BMA, low innervation of the $\mathrm{CeM}$, as well as dense inputs to the $\mathrm{CeL}$ and posterior MeA. Once again, direct innervation of the hippocampus was minimal.

Structural summary-In order to compare synaptophysin-mCherry expression across cases, the serial rostral-caudal measurements were averaged for each case to provide a single percent area value for each structure. This value was then corrected for median case percent area to account for between-case variation in construct expression. The corrected synaptophysin-mCherry density for each case is displayed in Figure 9. The averaging of rostral-caudal gradients combined with normalization of the density measurement revealed that all 3 cases had similar synaptophysin-mCherry expression across the major limbic nuclei. Therefore, the 3 cases were averaged to provide mean synaptophysin-mCherry density for all areas assessed across all cases. These corrected mean densities were plotted in 
a rank format (Figure 10) to statistically compare the structural output of the IL across all regions assessed (Figure 11). There was a main effect of region $[\mathrm{F}(34,68)=7.26, \mathrm{p}<$ 0.0001] on synaptophysin-mCherry expression and Bonferonni post-hoc analysis yielded pvalues to generate a significance matrix. Comparisons of IL structural output indicated that regions such as the MD and $\mathrm{PH}$ received significantly more IL input than most other forebrain regions, while the VMH and ventral subiculum received significantly less. Further, many large heterogeneous structures were compared for within-structure weighting of IL input. For instance, the MD received significantly more innervation than the paratenial thalamus (PT), PVT, nucleus reunions (Re), or centromedial thalamus (CM) and the PH received significantly more input than the mPOA, MPN, AHN, PVN, LH, VMH, and DMH.

\section{Experiment 3: optogenetic efficacy and specificity}

Optogenetic activation-In order to quantify the functional projections of the IL and to examine structure/function relationships, IL projection neurons were optogenetically activated followed by quantification of downstream immediate-early gene expression. Efficacy and selectivity of the approach was verified histochemically. Rats injected with either CAMKIIa-ChR2-YFP $(n=5)$ or CAMKIIa-YFP $(n=3)$ received pulsatile blue light stimulation ( $3 \mathrm{~mW}, 20 \mathrm{~Hz}, 5 \mathrm{~ms}$; Warden et al. 2012) for 5 minutes followed by assessment of Fos protein expression (Figure 12). Fos expression was apparent only in the ChR2expressing cells, indicating efficacy of stimulation parameters and ChR2 to induce cellular activation. To investigate CaMKIIa promotor selectivity, tissue from rats injected with CAMKIIa-YFP $(n=3)$ was analyzed for colocalization with the GABAergic marker GAD67 (Figures 13A and 13B). As the primary cortical output, CaMKIIa-positive cells should be almost exclusively glutamatergic and minimally colocalize with interneuronal GABAergic cells. Quantification of GAD-positive neurons and their colabeling with YFP indicated that there was some off-target expression of the vector (approximately $24 \%$ of GAD-positive cells also expressed YFP). However, as GAD-positive cells only represent $12-15 \%$ of the neurons in mPFC (McKlveen et al. 2015, 2016), the IL stimulation was predominantly, but not exclusively, glutamatergic. The placement of bilateral optic fiber tips from all animals included in the analysis $(\mathrm{N}=8)$ is depicted in Figure $13 \mathrm{C}$ relative to AAV injections. Although there was viral spread outside the IL, all fiber tips terminated within the IL, suggesting that the ventral-projecting optical stimulation was IL-predominant.

\section{Experiment 4: functional connectivity}

Fra immunoreactivity: mPFC_Immunoperoxidase labeling of Fra was used to quantify downstream functional activation following IL stimulation. While a c-Fos-specific antibody was used to verify optogenetic parameters (Figure 12), the Fra antibody was chosen to include as many acutely-activated cells as possible within the heterogeneous cell populations analyzed. Representative images of Fra expression in the mPFC of YFP- and ChR2-treated rats (Figure 14A and 14B) indicate efficacy of stimulation to induce cellular activation. The number of Fra-positive cells was quantified bilaterally in the rostral, middle, and caudal portions of each structure in YFP $(n=3)$ and $\operatorname{ChR} 2(n=5)$ rats. The means for each bregma level are shown as well as the average cellular activation across the total area assessed (Figure 14C and 14D). Compared to YFP, ChR2 led to greater light-evoked responses 
throughout the entire IL [t(6) $=2.61, \mathrm{p}=0.04]$. IL stimulation also significantly increased activation of the PL in ChR2-treated rats [t(6) $=2.59, \mathrm{p}=0.04]$.

NAC and BST-Representative images of Fra immunoreactivity from YFP- and ChR2treated rats illustrate cellular activation in the posterior BST (Figure 15A and 15B). Light administration into the IL did not produce significant differences in cellular activation in the NAc generally (Figure 16A) $[\mathrm{t}(6)=1.02, \mathrm{p}=0.35]$, nor the core (Figure 16B) $[\mathrm{t}(6)=0.38, \mathrm{p}$ $=0.71]$ or shell (Figure 16C) $[\mathrm{t}(6)=1.32, \mathrm{p}=0.23]$ specifically. Given the consistently dense expression of synaptophysin-mCherry throughout the BST in experiment 1 , it is of note that there were no changes in cellular activation in any BST subregion sampled. The anterior nuclei did not exhibit changes in Fra density (Figure 16D) [t(6) $=0.37, \mathrm{p}=0.73$ ]. Individual assessment of the adBST (dorsal portion of the am nucleus), avBST (dm, fu, and ventral portion of the am), and alBST (al, ov, and juxtacapsular [ju] nuclei) also did not identify differences in neuronal activation. The posterior nuclei did not yield differences in activation following IL stimulation (Figure 16E) [t(6) $=0.34, \mathrm{p}=0.75]$. The individual posterior subregions were further divided ([pr] and [if, tr]) and did not produce differences in Fra expression.

Hypothalamus-Representative images of hypothalamic Fra immunoreactivity in the region of the $\mathrm{PH}$ and $\mathrm{LH}$ are depicted in Figure 17A and 17B. Optogenetic activation of the IL produced heterogeneous responses in the diverse subregions of the hypothalamus. Optic stimulation of IL increased activation of the mPOA in ChR2-expressing rats $(n=5)$ compared to YFP controls $(\mathrm{n}=3$ ) (Figure 18A) [ $\mathrm{t}(6)=2.47, \mathrm{p}=0.04]$. IL stimulation did not alter total Fra expression in the MPN (Figure 18B) [t $(6)=0.53, \mathrm{p}=0.61]$; however, activation was significantly decreased in the rostral MPN $[\mathrm{t}(6)=4.08, \mathrm{p}=0.02]$. Stimulation of the IL also decreased activation of the AHN (Figure 18C) [t $(6)=3.14, \mathrm{p}=0.02]$. IL activation did not affect Fra-positive cell counts in the PVN (Figure 18D) $[\mathrm{t}(6)=2.05, \mathrm{p}=$ 0.08], LH (Figure 18E) [t(6) $=1.04, \mathrm{p}=0.34$ ], VMH (Figure 18F) [t(6) $=1.18, \mathrm{p}=0.28$ ], or $\mathrm{DMH}$ (Figure $18 \mathrm{G}$ ) $[\mathrm{t}(6)=1.12, \mathrm{p}=0.30]$. In contrast, IL illumination significantly increased Fra-immunoreactivity in the PH (Figure 18H) [t(6) $=6.22, \mathrm{p}=0.0008]$, most prominently in the rostral and middle portions.

Thalamus-Representative images of Fra immunoreactivity are depicted for the dorsal and medial aspects of the thalamus (Figure 19A and 19B). Stimulation of the IL in ChR2expressing rats $(\mathrm{n}=5)$ increased activity in multiple nuclei of the thalamus compared to YFP $(n=3)$. While there was no effect on total activation across all PT levels sampled (Figure 20A) $[\mathrm{t}(6)=0.40, \mathrm{p}=0.70]$, the caudal PT exhibited a significant increase in Fra expression [t(6) $=2.50, \mathrm{p}=0.04]$. The PVT (Figure 20B) $[\mathrm{t}(6)=2.89, \mathrm{p}=0.03]$ and MD (Figure 20D) $[\mathrm{t}(6)=2.50, \mathrm{p}=0.04]$ both showed significant functional connectivity with IL. In contrast, the $\operatorname{Re}$ (Figure 20C) $[\mathrm{t}(6)=0.76, \mathrm{p}=0.48]$ and $\mathrm{CM}$ (Figure 20E) $[\mathrm{t}(6)=1.45, \mathrm{p}$ $=0.19]$ regions had variable expression of Fra in both the YFP and ChR2 animals and did not yield statistically significant differences. The epithalamic LHab was also assessed (Figure 20F) $[\mathrm{t}(6)=0.80, \mathrm{p}=0.45]$ but did not show differences in cellular activation. 
Amygdala-Fra expression is illustrated in representative images from the amygdala of animals receiving either YFP or ChR2 (Figure 21A and 21B). Optical stimulation of the IL in rats expressing $\mathrm{ChR} 2(\mathrm{n}=5)$ led to increased activation of multiple amygdala subnuclei relative to YFP controls $(n=3)$. Within the BLA (LA included), there were significant differences across multiple rostral-caudal regions (Figure 22A) $[\mathrm{t}(6)=5.98, \mathrm{p}=0.001]$. Activation of IL also increased Fra-positive cell density in the CeA across all areas sampled (Figure 22B) [t(6) $=2.73, \mathrm{p}=0.03$ ]. Interestingly, when separated into the $\mathrm{CeM}$ and $\mathrm{CeL}$ (lateral capsular included) the increased functional activation was specifically accounted for by the CeL (Figure 22C) $[\mathrm{t}(6)=2.72, \mathrm{p}=0.03]$. Stimulation of the IL increased functional activation of MeA (Figure 22D) [t(6) =7.22, $\mathrm{p}=0.0004]$ with the same gradient across the anterior and posterior portions of the nucleus that was observed in Experiment 1.

Hippocampus-Representative images of dorsal hippocampal Fra immunoreactivity are depicted in Figure 23A and 23B. Activation of IL did not alter Fra expression in the hippocampus (Figure 24A-D). The CA3 [ $\mathrm{t}(6)=0.01, \mathrm{p}=0.99]$, CA1 [ $\mathrm{t}(6)=1.29, \mathrm{p}=0.24]$, $\mathrm{DG}[\mathrm{t}(6)=0.69, \mathrm{p}=0.51]$, and ventral hippocampus (vHipp) $[\mathrm{t}(6)=0.45, \mathrm{p}=0.67]$ all showed similar Fra densities in YFP $(n=3)$ and ChR2 $(n=5)$ rats.

Functional summary-The significant regional differences in Fra density between YFP and ChR2 are summarized in Table 1. Briefly, the PL, mPOA, PH, PT, PVT, MD, BLA, $\mathrm{CeA}$, and MeA all showed significant increases in neuronal activation, while MPN and AHN had decreases. To account for differences in Fra expression across regions, the percent change in neuronal activation relative to YFP controls was calculated for $\mathrm{ChR} 2$ rats. The percent change for each region was plotted in a ranked mean format (Figure 25) to statistically compare the functional output of the IL across all regions assessed (Figure 26). There was a main effect of region $[\mathrm{F}(26,105)=3.60, \mathrm{p}<0.0001]$ on percent change in neuronal activation and Bonferroni post-hoc analysis yielded $\mathrm{p}$ values to generate a significance matrix. This comparison of IL functional output indicated that regions with dense IL structural innervation, such as the $\mathrm{MD}$ and $\mathrm{PH}$, had significantly greater activation as a function of IL stimulation. However, numerous regions that significantly differed from YFP in terms of Fra density only showed modest increases in percent change (PVT, BLA, CeL), while other nuclei that exhibited no differences from YFP in Fra density showed higher levels of mean percent change (LH, CM). The later effect appears to be driven by a small number of highly-responsive animals. Within-structure comparisons were made to discern significant differences in functional connectivity. For instance, the PH shows significantly more IL functional connectivity than the AHN, PVN, or DMH, while the AHN has significantly less than mPOA, LH, and PH.

\section{Discussion}

A novel viral genetic approach allowed the systematic quantification of IL structural output, yielding a comprehensive map of IL presynaptic density throughout and within the major nuclei of the limbic forebrain. The weighting of this input was statistically compared across regions to determine the nuclei with the greatest innervation density. Specifically targeting a fluorescent reporter to the presynaptic terminal allowed quantification of inputs without including fibers of passage. Additionally, cell-type specific optogenetics permitted temporal 
control of neural activity within IL glutamate neurons. Cellular activation was quantified to determine functional connectivity of IL projections via the induction of the Fra family of immediate-early genes. Collectively, these two approaches identified a relatively small number of structures with high levels of IL presynaptic input that also showed robust Fra expression after IL stimulation. These primarily included the MD, $\mathrm{PH}$, posterior MeA, and $\mathrm{CeL}$. There were several nuclei with little structural or functional input from the IL. These included multiple subfields of the NAc and hippocampus, as well as the VMH and PVN. Perhaps most surprising were those nuclei that received dense structural input but did not yield significant increases in Fra expression. For example, the subregions of the BST received consistently robust IL input, yet did not exhibit functional changes following IL stimulation.

Compared to previous measures of IL structural efferents using Phaseolus vulgaris leucoagglutinin, our data are largely corroborative, showing IL projections to the limbic thalamus, BST, amygdala, and hypothalamus (Hurley et al. 1991; Vertes 2004). The current analysis also yields a wealth of new data, primarily through identifying the quantifiable differences between subregions of structures. For instance, previous studies did not report major differences between IL innervation of the $\mathrm{LH}, \mathrm{DMH}$, and $\mathrm{PH}$, while our results indicate IL connectivity with the PH is significantly greater than the LH or DMH. Additionally, determining coordinate-specific connectivity across the rostral-caudal extension of nuclei generated a projection map, or 'projectome', that allows for specific and accurate targeting of IL output circuits for monitoring and/or manipulation studies. Importantly, activation of IL output provided the ability to map functional changes in activity of downstream nuclei, yielding unprecedented comparisons of structural and functional connectivity. The current study also sheds light on the nature of IL inputs to the NAc, an area with mixed findings in the literature. Some have reported IL innervation of the NAc (Hurley et al. 1991; Thompson and Swanson 2010); however, our current data and those of Vertes (Vertes 2004) suggest that mPFC inputs to the NAc are not mediated by the IL and likely arise from more dorsal regions in the PL. Alternatively, the current findings that IL projections do not directly innervate the hippocampus, VMH, or PVN are in agreement with previous reports (Hurley et al. 1991; Vertes 2004).

There are several technical considerations that should be taken into account when interpreting the current findings. For structural analyses, there was limited spread of the lentiviral injections in the cases presented. As the cases with larger injections consistently spread dorsally into the PL, the injections that remained in the IL were largely in the deep layers of the mid IL, without full spread through the superficial layers or caudal IL. Consequently, there may be IL cell groups with connectivity not represented in the structural analysis. Another consideration related to the lentiviral approach is that the synaptic targeting of mCherry did not fill the cell bodies of transfected neurons. While it was possible to examine protein conjugate expression in the transfected cells to determine injection localization, a second nuclear-localized reporter would improve injection visualization. Importantly, the downstream sites analyzed do not represent the totality of IL projections. As the focus of this study was descending projections to the limbic forebrain involved in homeostatic and behavioral adaptation, other important cortical-cortical and corticalbrainstem circuits were not explored. Several analyzed regions exhibited minimal 
innervation, yet the sensitivity of presynaptic reporting and high-magnification optical sectioning quantified these minor projections. Generally, mean percent area values below $0.05 \%$ were considered negligible. The structural analysis also had restricted statistical power based on the sample size; thus, there are interpretative limitations when differences in regional innervation are not statistically significant.

Regarding functional analyses, it is important to note that the absence of increased Fra expression does not confirm a lack of neuronal activation. Immediate-early gene expression varies across cell types and brain regions with numerous cellular response systems that can be activated independent of the Fra family. Accordingly, negative results from the functional connectivity analysis do not preclude IL functional interactions with the cell groups analyzed. Furthermore, while the synaptophysin-mCherry fusion protein was used to quantify monosynaptic connectivity, the timing of protein expression after optogenetic activation did not restrict the functional analysis to monosynaptic connections. Therefore, changes in Fra expression may result from transsynaptic activation/inhibition. In fact, areas showing decreased Fra (MPN and AHN) likely result from transsynaptic inhibition; although, this could also relate to the postsynaptic neurochemistry of these regions. Differences in imaging resolution between structural and functional studies represent an additional limitation of the current experiments. Brightfield imaging of immunoperoxidase labeling in the functional analyses could not be done with the same resolution as the genetically-encoded fluorescence in the structural analyses. Consequently, there were regions that could not be reliably separated by blinded-observers in the functional analysis (e.g. dmBST and fuBST, LA and BLA, etc.) and a small number that were not differentiated in brightfield due to a lack of landmarks (cortical amygdala and BMA). Thus, the functional analysis contained fewer individual regions than the structural analysis.

Given the essential role of mPFC in mood and cognition, further investigation of specific output circuitry is needed. Patients with mPFC lesions exhibit impaired decision-making leading to behavior based on insensitivity to future consequences (Bechara et al. 2000). Similarly, chronic stress impairs executive functions such as working memory and cognitive flexibility in rodents (Holmes and Wellman 2009), presumably through altering mPFC neuronal function and morphology (Radley et al. 2004, 2006, 2008; Cook and Wellman 2004). The rodent IL also mediates chronic stress effects on hypothalamic-pituitary-adrenal axis reactivity (Myers et al. 2017) and glucocorticoid negative-feedback (Myers et al. 2012; McKlveen et al. 2013, 2016). Interactions between stress and the IL may be important for affective pathology; in fact, activity of the IL is critical for fear extinction, an effect hypothesized to be mediated by innervation of intercalated cells that inhibit the CeM (Sotres-Bayon and Quirk 2010). Our data suggest that IL interactions with amygdala fear and anxiety centers are extensive and complex. The IL had considerable efferent innervation of the BMA and LA, both associated with learned fear (Erlich et al. 2012; Adhikari et al. 2015). Additionally, the CeL had high levels of structural and functional connectivity and has been shown to provide inhibitory innervation of the CeM to gate anxiety-like behavior (Tye et al. 2011).

Multiple autonomic functions have been attributed to the IL, with some considering the region a component of visceral cortex (Terreberry and Neafsey 1983; Frysztak and Neafsey 
1994). Numerous studies have utilized lesions or electrical stimulation of ventral mPFC to identify roles in the regulation of blood pressure, heart rate, and gastric function (HurleyGius and Neafsey 1986; Hardy and Holmes 1988; Myers 2017). The specific cellular and circuit interactions that mediate these effects are largely unknown. Hypothalamic projections outlined in the current study likely play a role, with the PH representing a primary candidate. This nucleus regulates heart rate and vasomotor responses (Lisa et al. 1989; Gao et al. 2016) and mediates neuroendocrine stress reactivity (Nyhuis et al. 2016; Myers et al. 2016a). We have previously shown that the IL targets intranuclear GABAergic neurons within the PH (Myers et al. 2016a), providing a potential mechanism for IL control of autonomic activity. Although the current analysis was focused on the limbic forebrain, IL projections to the brainstem are an important consideration for autonomic function (Rinaman 2011; Myers et al. 2016b). Retrograde tracer injections in the nucleus of the solitary tract densely label IL (Gabbott et al. 2005) and projections to monoaminergic nuclei have been described (Hurley et al. 1991; Vertes 2004), suggesting that IL-brainstem interactions are an area where future study is warranted.

Connectivity with the medial thalamic nuclei is a common criteria for comparative analysis of mPFC subregions (Uylings and van Eden 1990). Accordingly, there were dense projections to the MD and PVT. These are among the thalamic nuclei composing the limbic thalamus (Vertes et al. 2015), a network with reciprocal corticolimbic connectivity. Despite the level of interconnectedness between the limbic thalamus and $\mathrm{mPFC}$, the functional outcomes of this circuitry are not completely understood. The MD has been implicated in behavioral regulation and cognitive flexibility; furthermore, experiments using an asymmetric inactivation approach indicate that disruption of IL-MD communication induces perseverative behaviors (Block et al. 2007). Interestingly, the PVT is responsive to a variety of stressors, integrates stress chronicity, and interacts with structures that regulate motivation and mood (Hsu et al. 2014). Thus, the prominent IL structural and functional projections to the MD and PVT are likely critical components of the networks regulating affect and behavioral flexibility.

In conclusion, viral-mediated expression of a synaptic marker allowed the quantification of IL pre-synaptic terminals throughout the limbic forebrain. Moreover, optogenetic stimulation of IL projection neurons permitted the quantification of functional interactions. Collectively, this specific assessment of IL structural and functional projections forms the basis for data-driven analysis of descending corticolimbic networks.

\section{Acknowledgments}

AAV vectors were provided by the University of North Carolina Vector Core under material transfer agreement with Karl Deisseroth and Stanford University.

Funding: This work was supported by NIH grant K99/R00 HL122454 and an American Heart Association Fellowship to B. Myers, as well as NIH grants R01 MH049698 and R01 MH101729 to J.P. Herman. T. Wallace received support from the Colorado State University Molecular, Cellular and Integrative Neuroscience program.

\section{Abbreviation index}

third ventricle 


\begin{tabular}{|c|c|}
\hline A488 & Alexa Fluor 488 \\
\hline aBST & anterior BST \\
\hline $\mathbf{A A V}$ & adeno-associated virus \\
\hline $\mathbf{A H N}$ & anterior hypothalamic nucleus \\
\hline AV & anteroventral thalamus \\
\hline BA25 & Brodmann area 25 \\
\hline BLA & basolateral amygdala \\
\hline BMA & basomedial amygdala \\
\hline BST & bed nucleus of the stria terminalis \\
\hline BSTal & anterolateral BST \\
\hline BSTald & dorsal portion of anterolateral BST \\
\hline BSTalv & ventral portion of anterolateral BST \\
\hline BSTam & anteromedial BST \\
\hline BSTamd & dorsal portion of anteromedial BST \\
\hline BSTamv & ventral portion of anteromedial BST \\
\hline BSTfu & fusiform BST \\
\hline BSTif & interfascicular BST \\
\hline BSTov & oval BST \\
\hline BSTpr & principal BST \\
\hline BSTtr & transverse BST \\
\hline CA1 & cornu ammonis field 1 \\
\hline CA3 & cornu ammonis field 3 \\
\hline CaMKIIa & $\mathrm{Ca}^{2+} /$ calmodulin-dependent protein kinase II alpha \\
\hline cc & corpus callosum \\
\hline $\mathbf{C e A}$ & central nucleus of the amygdala \\
\hline CeL & lateral subdivision of central amygdala \\
\hline CeM & medial subdivision of central amygdala \\
\hline ChR2 & channelrhodopsin-2 \\
\hline CM & centromedial thalamus \\
\hline
\end{tabular}




\begin{tabular}{|c|c|}
\hline CoA & cortical amygdala \\
\hline DAPI & 4',6-Diamidino-2-Phenylindole \\
\hline DG & dentate gyrus \\
\hline DMH & dorsomedial hypothalamus \\
\hline fa & corpus callosum anterior forceps \\
\hline $\mathbf{f x}$ & fornix \\
\hline Fra & fos-related antigen \\
\hline GAD67 & glutamic acid decarboxylase, $67 \mathrm{kDa}$ isoform \\
\hline IHC & immunohistochemistry \\
\hline IL & infralimbic cortex \\
\hline LA & lateral amygdala \\
\hline $\mathbf{L H}$ & lateral hypothalamus \\
\hline LHab & lateral habenula \\
\hline $\mathbf{m C h}$ & mCherry \\
\hline MD & medialdorsal thalamus \\
\hline MDD & major depressive disorder \\
\hline MeA & medial amygdala \\
\hline MeAad & anterodorsal $\mathrm{MeA}$ \\
\hline MeApd & posterodorsal MeA \\
\hline MeApv & posteroventral MeA \\
\hline MOC & Manders' overlap coefficient \\
\hline mPFC & medial prefrontal cortex \\
\hline MPN & medial preoptic nucleus \\
\hline MPOA & medial preoptic area \\
\hline mtt & mammillothalamic tract \\
\hline NAc & nucleus accumbens \\
\hline NAcC & NAc core \\
\hline NAcS & NAc shell \\
\hline NeuN & neuronal nuclear protein \\
\hline
\end{tabular}




$\begin{array}{ll}\text { ot } & \text { optic tract } \\ \text { pBST } & \text { posterior BST } \\ \text { PH } & \text { posterior hypothalamic nucleus } \\ \text { PL } & \text { prelimbic cortex } \\ \text { PT } & \text { paratenial thalamus } \\ \text { PVN } & \text { paraventricular nucleus of the hypothalamus } \\ \text { PVT } & \text { paraventricular thalamus } \\ \text { Re } & \text { nucleus reunions } \\ \text { Sph } & \text { synaptophysin } \\ \text { sm } & \text { stria medullaris } \\ \text { St } & \text { stria terminalis } \\ \text { VMH } & \text { ventromedial hypothalamus } \\ \text { vSub } & \text { ventral subiculum } \\ \text { YFP } & \text { yellow fluorescent protein }\end{array}$

\section{References}

Adhikari A, Lerner TN, Finkelstein J, et al. (2015) Basomedial amygdala mediates top-down control of anxiety and fear. Nature 527:179-185. [PubMed: 26536109]

Bechara A, Tranel D, Damasio H (2000) Characterization of the decision-making deficit of patients with ventromedial prefrontal cortex lesions. Brain 123 (Pt 1:2189-202) [PubMed: 11050020]

Block AE, Dhanji H, Thompson-Tardif SF, Floresco SB (2007) Thalamic-Prefrontal Cortical-Ventral Striatal Circuitry Mediates Dissociable Components of Strategy Set Shifting. Cereb Cortex 17:1625-1636. [PubMed: 16963518]

Budzikowski AS, Vahid-Ansari F, Leenen FH (1998) Chronic activation of brain areas by high-sodium diet in Dahl salt-sensitive rats. Am J Physiol 274:H2046-52 [PubMed: 9841531]

Carvalho-Netto EF, Myers B, Jones K, et al. (2011) Sex differences in synaptic plasticity in stressresponsive brain regions following chronic variable stress. Physiol Behav 104:242-247 [PubMed: 21315096]

Cook SC, Wellman CL (2004) Chronic stress alters dendritic morphology in rat medial prefrontal cortex. J Neurobiol 60:236-48. [PubMed: 15266654]

Covington HE, Lobo MK, Maze I, et al. (2010) Antidepressant Effect of Optogenetic Stimulation of the Medial Prefrontal Cortex. J Neurosci 30:16082-16090. [PubMed: 21123555]

Damasio AR, Everitt BJ, Bishop D (1996) The Somatic Marker Hypothesis and the Possible Functions of the Prefrontal Cortex [and Discussion]. Philos Trans R Soc B Biol Sci 351:1413-1420.

Davern PJ, Head GA (2007) Fos-Related Antigen Immunoreactivity After Acute and Chronic Angiotensin II-Induced Hypertension in the Rabbit Brain. Hypertension 49:1170-1177. [PubMed: 17339536]

de Almeida LP, Zala D, Aebischer P, Déglon N (2001) Neuroprotective Effect of a CNTF-Expressing Lentiviral Vector in the Quinolinic Acid Rat Model of Huntington's Disease. Neurobiol Dis 8:433-446. [PubMed: 11442352] 
Drevets WC, Price JL, Furey ML (2008) Brain structural and functional abnormalities in mood disorders: implications for neurocircuitry models of depression. Brain Struct Funct 213:93-118. [PubMed: 18704495]

Drevets WC, Price JL, Simpson JR, et al. (1997) Subgenual prefrontal cortex abnormalities in mood disorders. Nature 386:824-7. [PubMed: 9126739]

Dum RP, Levinthal DJ, Strick PL (2016) Motor, cognitive, and affective areas of the cerebral cortex influence the adrenal medulla. Proc Natl Acad Sci U S A 113:9922-7. [PubMed: 27528671]

Erlich JC, Bush DEA, LeDoux JE (2012) The role of the lateral amygdala in the retrieval and maintenance of fear-memories formed by repeated probabilistic reinforcement. Front Behav Neurosci 6:16. [PubMed: 22514524]

Ferrer I, Olivé M, Ribera J, Planas AM (1996) Naturally Occurring (Programmed) and Radiationinduced Apoptosis are Associated with Selective c-Jun Expression in the Developing Rat Brain. Eur J Neurosci 8:1286-1298. [PubMed: 8752598]

Frysztak RJ, Neafsey EJ (1994) The effect of medial frontal cortex lesions on cardiovascular conditioned emotional responses in the rat. Brain Res 643:181-93 [PubMed: 8032913]

Fuchikami M, Thomas A, Liu R, et al. (2015) Optogenetic stimulation of infralimbic PFC reproduces ketamine's rapid and sustained antidepressant actions. Proc Natl Acad Sci U S A 112:8106-11. [PubMed: 26056286]

Gabbott PLA, Warner TA, Jays PRL, et al. (2005) Prefrontal cortex in the rat: projections to subcortical autonomic, motor, and limbic centers. J Comp Neurol 492:145-77. [PubMed: 16196030]

Gao H-R, Zhuang Q-X, Li B, et al. (2016) Corticotropin releasing factor excites neurons of posterior hypothalamic nucleus to produce tachycardia in rats. Sci Rep 6:20206. [PubMed: 26831220]

Grillo CA, Piroli GG, Lawrence RC, et al. (2015) Hippocampal Insulin Resistance Impairs Spatial Learning and Synaptic Plasticity. Diabetes 64:3927-3936. [PubMed: 26216852]

Hamani C, Diwan M, Macedo CE, et al. (2010) Antidepressant-like effects of medial prefrontal cortex deep brain stimulation in rats. Biol Psychiatry 67:117-24. [PubMed: 19819426]

Hardy SG, Holmes DE (1988) Prefrontal stimulus-produced hypotension in rat. Exp brain Res 73:24955 [PubMed: 3215302]

Holmes A, Wellman CL (2009) Stress-induced prefrontal reorganization and executive dysfunction in rodents. Neurosci Biobehav Rev 33:773-783. [PubMed: 19111570]

Hsu DT, Kirouac GJ, Zubieta J-K, Bhatnagar S (2014) Contributions of the paraventricular thalamic nucleus in the regulation of stress, motivation, and mood. Front Behav Neurosci 8:73. [PubMed: 24653686]

Hurley-Gius KM, Neafsey EJ (1986) The medial frontal cortex and gastric motility: microstimulation results and their possible significance for the overall pattern of organization of rat frontal and parietal cortex. Brain Res 365:241-8 [PubMed: 3947992]

Hurley KM, Herbert H, Moga MM, Saper CB (1991) Efferent projections of the infralimbic cortex of the rat. J Comp Neurol 308:249-276 [PubMed: 1716270]

Jones KR, Myers B, Herman JP (2011) Stimulation of the prelimbic cortex differentially modulates neuroendocrine responses to psychogenic and systemic stressors. Physiol Behav 104:266-271 [PubMed: 21443894]

Krause EG, de Kloet AD, Scott KA, et al. (2011) Blood-borne angiotensin II acts in the brain to influence behavioral and endocrine responses to psychogenic stress. J Neurosci 31:15009-15. [PubMed: 22016534]

Laurent V, Westbrook RF (2009) Inactivation of the infralimbic but not the prelimbic cortex impairs consolidation and retrieval of fear extinction. Learn Mem 16:520-529. [PubMed: 19706835]

Lisa M, Marmo E, Wible JH, Jr, DiMicco JA (1989) Injection of muscimol into posterior hypothalamus blocks stress-induced tachycardia. Am J Physiol 257:R246-51 [PubMed: 2750964]

Manders EM, Stap J, Brakenhoff GJ, et al. (1992) Dynamics of three-dimensional replication patterns during the S-phase, analysed by double labelling of DNA and confocal microscopy. J Cell Sci 103 (Pt 3):857-62 [PubMed: 1478975] 
Mayberg HS, Liotti M, Brannan SK, et al. (1999) Reciprocal limbic-cortical function and negative mood: converging PET findings in depression and normal sadness. Am J Psychiatry 156:675-82. [PubMed: 10327898]

Mayberg HS, Lozano AM, Voon V, et al. (2005) Deep brain stimulation for treatment-resistant depression. Neuron 45:651-660. [PubMed: 15748841]

McClung CA, Ulery PG, Perrotti LI, et al. (2004) DeltaFosB: a molecular switch for long-term adaptation in the brain. Brain Res Mol Brain Res 132:146-54. [PubMed: 15582154]

McKlveen JM, Morano RL, Fitzgerald M, et al. (2016) Chronic stress increases prefrontal inhibition: a mechanism for stress-induced prefrontal dysfunction. Biol Psychiatry 80:754-764. [PubMed: 27241140]

McKlveen JM, Myers B, Flak JN, et al. (2013) Role of Prefrontal Cortex Glucocorticoid Receptors in Stress and Emotion. Biol Psychiatry 74:672-9 [PubMed: 23683655]

McKlveen JM, Myers B, Herman JP (2015) The medial prefrontal cortex: coordinator of autonomic, neuroendocrine and behavioural responses to stress. J Neuroendocrinol 27:446-56. [PubMed: 25737097]

Myers B (2017) Corticolimbic regulation of cardiovascular responses to stress. Physiol Behav 172:4959. [PubMed: 27793557]

Myers B, Carvalho-Netto E, Wick-Carlson D, et al. (2016a) GABAergic Signaling within a LimbicHypothalamic Circuit Integrates Social and Anxiety-Like Behavior with Stress Reactivity. Neuropsychopharmacology 41:1530-1539. [PubMed: 26442601]

Myers B, Dolgas CM, Kasckow J, et al. (2014a) Central stress-integrative circuits: forebrain glutamatergic and GABAergic projections to the dorsomedial hypothalamus, medial preoptic area, and bed nucleus of the stria terminalis. Brain Struct Funct 219:1287-303. [PubMed: 23661182]

Myers B, McKlveen JM, Herman JP (2012) Neural Regulation of the Stress Response: The Many Faces of Feedback. Cell Mol Neurobiol 32:683-694

Myers B, McKlveen JM, Herman JP (2014b) Glucocorticoid actions on synapses, circuits, and behavior: implications for the energetics of stress. Front Neuroendocrinol 35:180-96. [PubMed: 24361584]

Myers B, McKlveen JM, Morano R, et al. (2017) Vesicular Glutamate Transporter 1 Knockdown in Infralimbic Prefrontal Cortex Augments Neuroendocrine Responses to Chronic Stress in Male Rats. Endocrinology 158:3579-3591. [PubMed: 28938481]

Myers B, Scheimann JR, Franco-Villanueva A, Herman JP (2016b) Ascending mechanisms of stress integration: Implications for brainstem regulation of neuroendocrine and behavioral stress responses. Neurosci Biobehav Rev 74:366-375. [PubMed: 27208411]

Nyhuis TJ, Masini CV, Day HEW, Campeau S (2016) Evidence for the Integration of Stress-Related Signals by the Rostral Posterior Hypothalamic Nucleus in the Regulation of Acute and Repeated Stress-Evoked Hypothalamo-Pituitary-Adrenal Response in Rat. J Neurosci 36:795-805. [PubMed: 26791210]

Paxinos G, Watson C (1998) The rat brain in stereotaxic coordinates. Elsevier

Radley JJ, Rocher AB, Miller M, et al. (2006) Repeated stress induces dendritic spine loss in the rat medial prefrontal cortex. Cereb Cortex 16:313-20. [PubMed: 15901656]

Radley JJ, Rocher AB, Rodriguez A, et al. (2008) Repeated stress alters dendritic spine morphology in the rat medial prefrontal cortex. J Comp Neurol 507:1141-1150 [PubMed: 18157834]

Radley JJ, Sisti HM, Hao J, et al. (2004) Chronic behavioral stress induces apical dendritic reorganization in pyramidal neurons of the medial prefrontal cortex. Neuroscience 125:1-6. [PubMed: 15051139]

Rinaman L (2011) Hindbrain noradrenergic A2 neurons: diverse roles in autonomic, endocrine, cognitive, and behavioral functions. Am J Physiol Regul Integr Comp Physiol 300:R222-35. [PubMed: 20962208]

Sierra-Mercado D, Padilla-Coreano N, Quirk GJ (2011) Dissociable roles of prelimbic and infralimbic cortices, ventral hippocampus, and basolateral amygdala in the expression and extinction of conditioned fear. Neuropsychopharmacology 36:529-38. [PubMed: 20962768]

Sotres-Bayon F, Quirk GJ (2010) Prefrontal control of fear: more than just extinction. Curr Opin Neurobiol 20:231-5. [PubMed: 20303254] 
Swanson LW (2004) Brain Maps: Structure of the Rat Brain (3rd edition)

Team R (2014) R: A language and environment for statistical computing R Foundation for Statistical Computing, Vienna, Austria. 2013

Terreberry RR, Neafsey EJ (1983) Rat medial frontal cortex: a visceral motor region with a direct projection to the solitary nucleus. Brain Res 278:245-249. [PubMed: 6315155]

Thompson RH, Swanson LW (2010) Hypothesis-driven structural connectivity analysis supports network over hierarchical model of brain architecture. Proc Natl Acad Sci U S A 107:15235-9. [PubMed: 20696892]

Tye KM, Prakash R, Kim S-Y, et al. (2011) Amygdala circuitry mediating reversible and bidirectional control of anxiety. Nature 471:358-62. [PubMed: 21389985]

Uylings HB, van Eden CG (1990) Qualitative and quantitative comparison of the prefrontal cortex in rat and in primates, including humans. Prog Brain Res 85:31-62 [PubMed: 2094901]

Uylings HBM, Groenewegen HJ, Kolb B (2003) Do rats have a prefrontal cortex? Behav Brain Res 146:3-17 [PubMed: 14643455]

Vertes RP (2004) Differential projections of the infralimbic and prelimbic cortex in the rat. Synapse 51:32-58 [PubMed: 14579424]

Vertes RP, Linley SB, Hoover WB (2015) Limbic circuitry of the midline thalamus. Neurosci Biobehav Rev 54:89-107. [PubMed: 25616182]

Warden MR, Selimbeyoglu A, Mirzabekov JJ, et al. (2012) A prefrontal cortex-brainstem neuronal projection that controls response to behavioural challenge. Nature 492:428-32. [PubMed: 23160494]

Wickham H (2009) Ggplot2: elegant graphics for data analysis. Springer

Wiedenmann B, Franke WW (1985) Identification and localization of synaptophysin, an integral membrane glycoprotein of Mr 38,000 characteristic of presynaptic vesicles. Cell 41:1017-28 [PubMed: 3924408]

Wood JN, Grafman J (2003) Human prefrontal cortex: processing and representational perspectives. Nat Rev Neurosci 4:139-147. [PubMed: 12563285] 


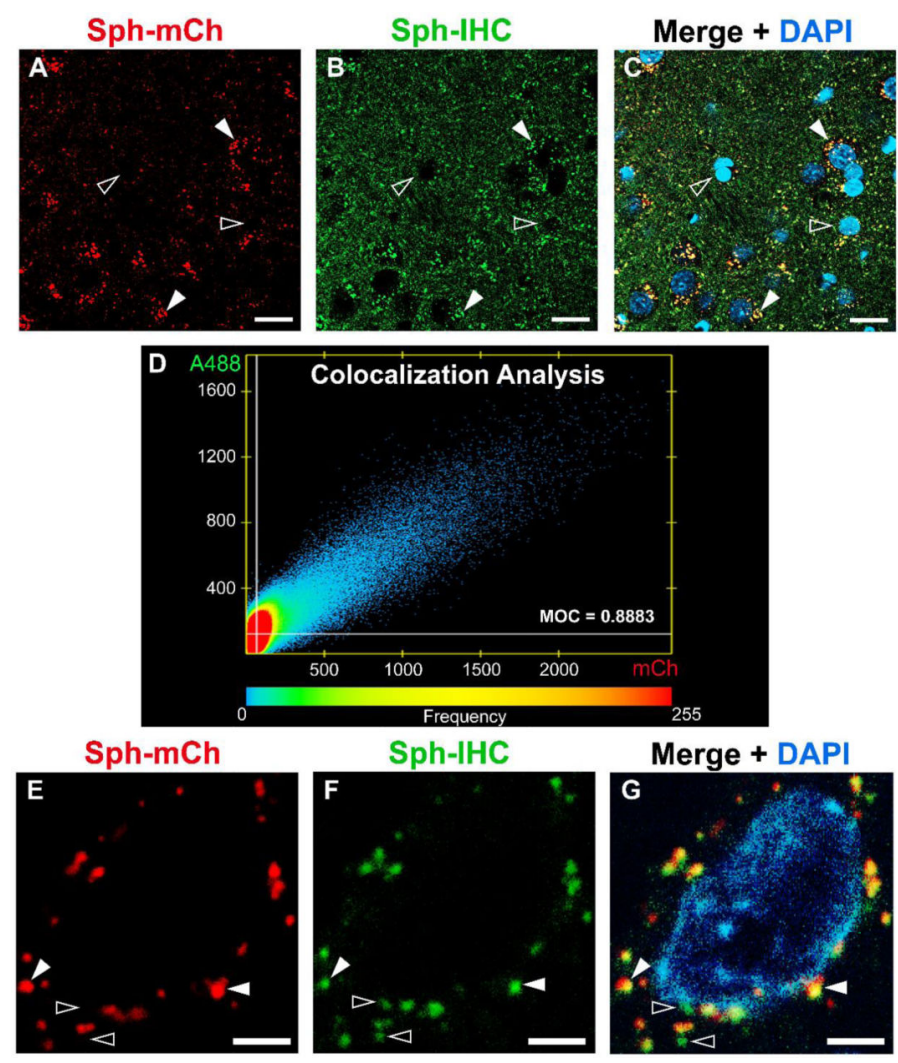

Figure 1.

Injection of a lentiviral-packaged vector coding for synaptophysin-mCherry into the IL produced mCherry (red) expression in the MD (A). Immunohistochemical labeling of synaptophysin (B; green) was used to verify construct efficacy $(\mathbf{C})$. Nuclei were counterstained with DAPI (blue). Colocalization analysis indicated high-levels of overlap between genetically-encoded synaptophysin and antibody-visualized synaptophysin (D). Viral injections in the IL led to mCherry (red) expression in the PH (E). Immunohistochemical labeling of synaptophysin $(\mathbf{F}$; green) was coincident $(\mathbf{G})$. Nuclei were counterstained with DAPI (blue). White-filled arrows point to examples of colocalization and unfilled arrows highlight single-labeled Sph-IHC. A488: Alexa Fluor 488; IHC: immunohistochemistry; mCh: mCherry; MOC: Manders' overlap coefficient; Sph: synaptophysin; Scale bars: A-C $20 \mu \mathrm{m}$; E-G $5 \mu \mathrm{m}$. 


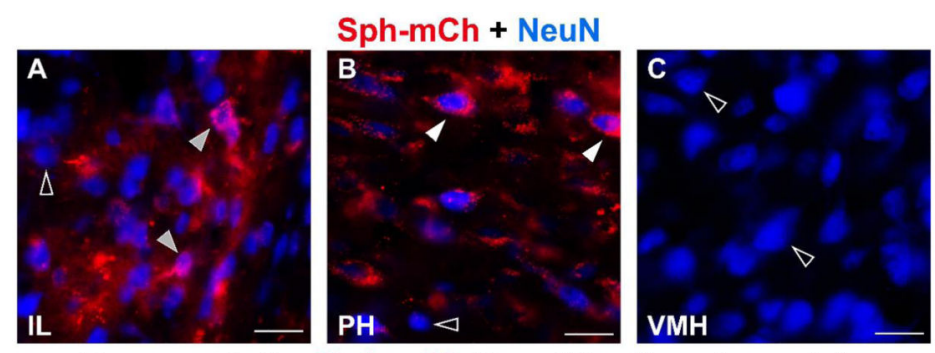

Representative Sph-mCh Quantification: Amygdala

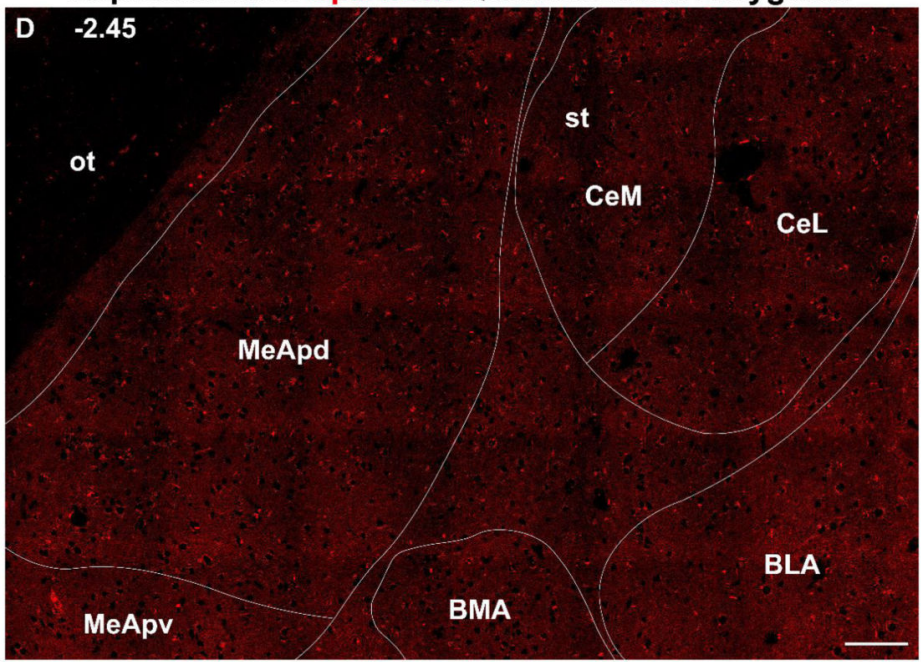

Figure 2.

Representative injection site for synaptophysin-mCherry (red) in the IL (A) with neurons visualized via NeuN labeling (blue). Synaptophysin-mCherry was expressed in the PH (B), a previously characterized target of IL. In contrast, there was little synaptophysin-mCherry in the $\mathrm{VMH}(\mathbf{C})$, a region reported to have minimal cortical input. Representative tiling of images from 40x objective used to quantify synaptophysin-mCherry (D; red) throughout the forebrain. Distance listed is caudal to bregma $(\mathrm{mm})$. Grey-filled arrows highlight examples of transfected IL neurons, white-filled arrows point to neurons receiving IL input, and unfilled arrows highlight neurons without mCherry. BLA: basolateral amygdala; BMA: basomedial amygdala; CeL: lateral subdivision of central amygdala; CeM: medial subdivision of central amygdala; IL: infralimbic cortex; mCh: mCherry; MeApd: posterodorsal medial amygdala; MeApv: posteroventral medial amygdala; ot: optic tract; NeuN: neuronal nuclear protein; PH: posterior hypothalamic nucleus; Sph: synaptophysin; st: stria terminalis; VMH: ventromedial hypothalamus; Scale bars: A-C 100 $\mu \mathrm{m}$; D $200 \mu \mathrm{m}$. 

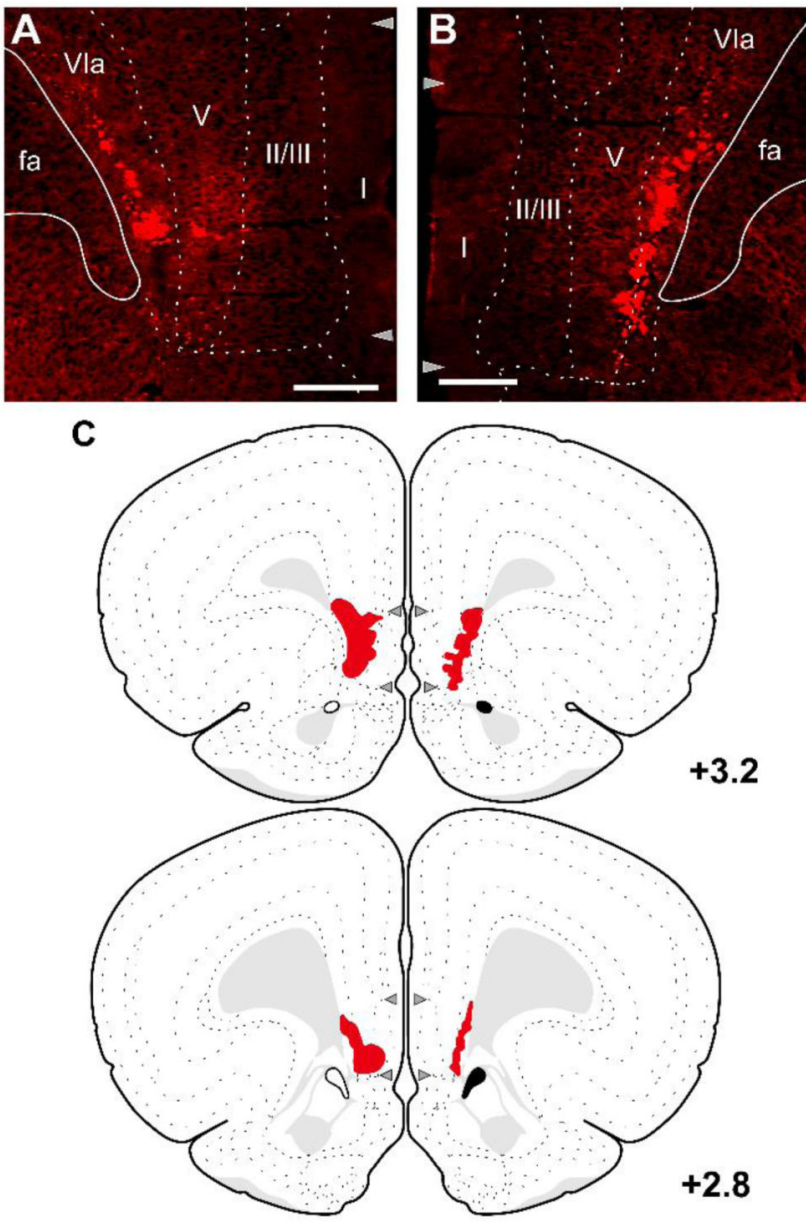

Figure 3.

Case 13 injection placement. Photomicrographs illustrate injection sites for synaptophysinmCherry (red) in the right IL (A) and left IL (B). The most rostral and caudal extents of the injections were mapped onto atlas templates (C; Swanson 2004). Distances listed are rostral to bregma $(\mathrm{mm})$ and Roman numerals indicate cortical layers. Grey arrows designate dorsal and ventral boundaries of IL. Scale bars: $200 \mu \mathrm{m}$. 


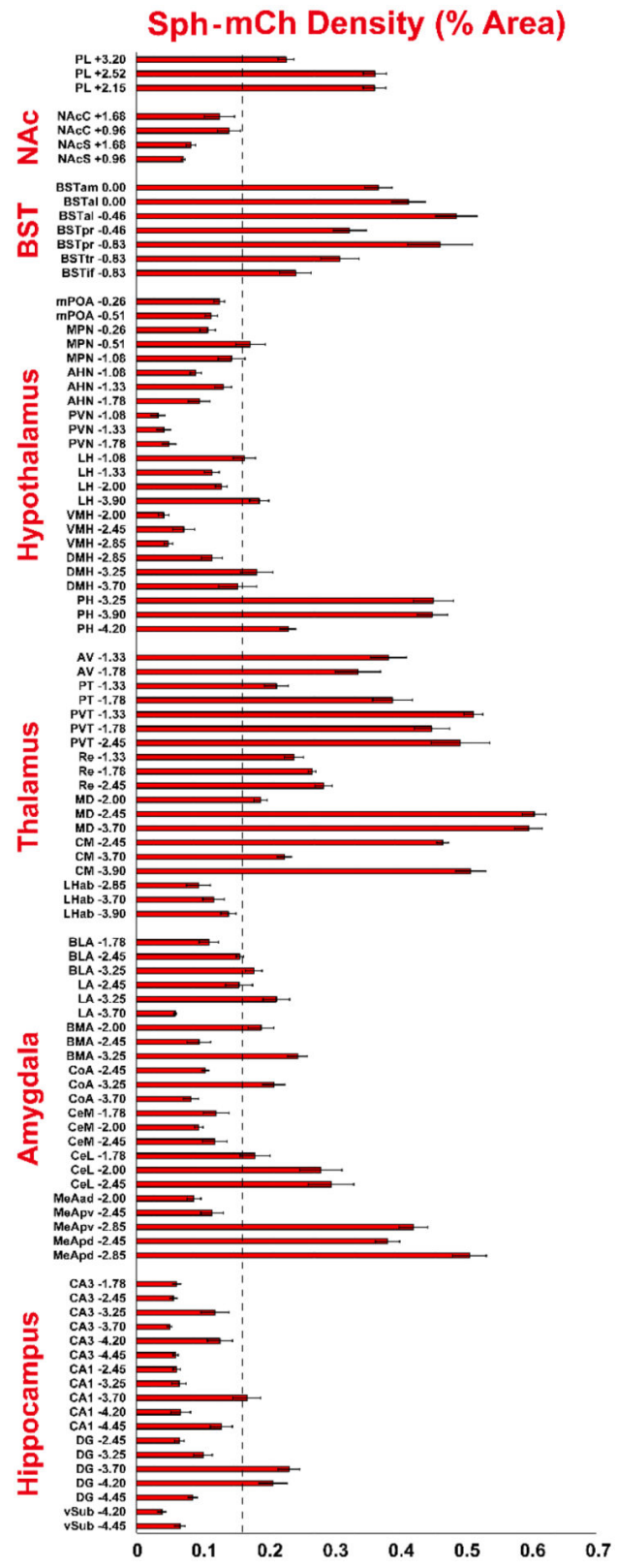

Figure 4.

Case 13 synaptophysin-mCherry expression (percent area) in the limbic forebrain. Values expressed are mean \pm SEM from serial high-magnification images. Dashed line represents case median percent density. Distances listed are relative to bregma. See index for abbreviations. 

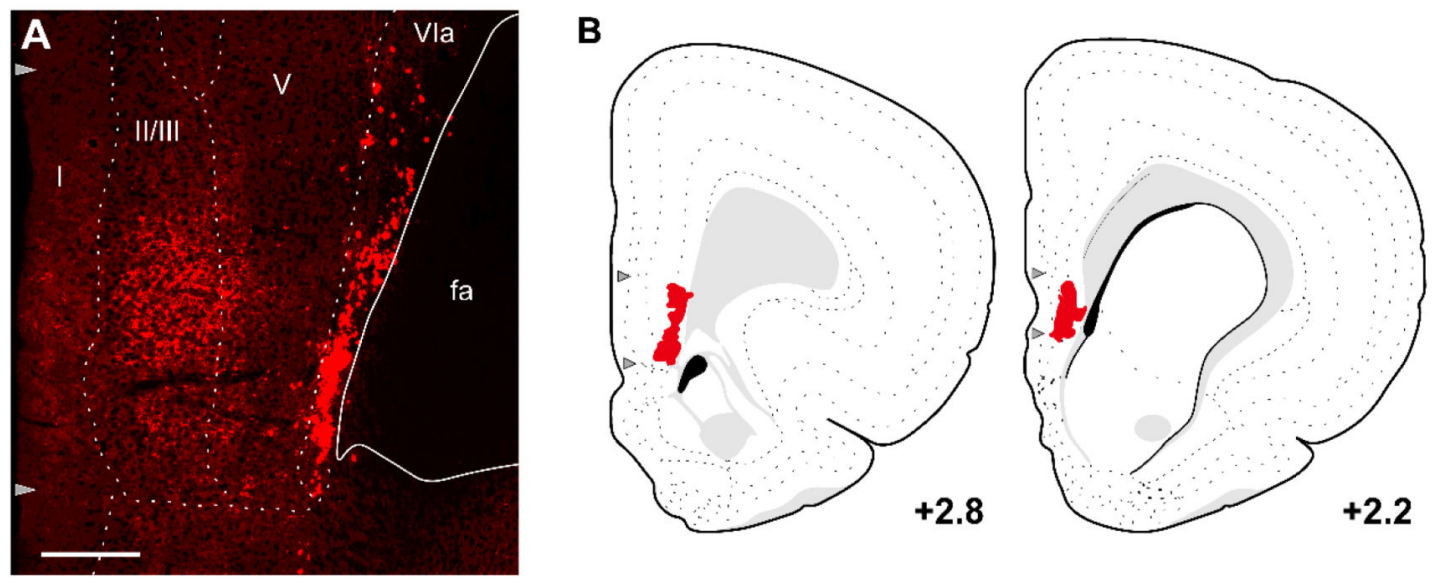

Figure 5.

Case 14 injection placement. Photomicrographic illustration of injection site for synaptophysin-mCherry (red) in the left IL (A). The most rostral and caudal extents of the injections were mapped onto atlas templates (B; Swanson 2004). Distances listed are rostral to bregma $(\mathrm{mm})$ and Roman numerals indicate cortical layers. Grey arrows indicate dorsal and ventral boundaries of IL. Scale bar: $200 \mu \mathrm{m}$. 


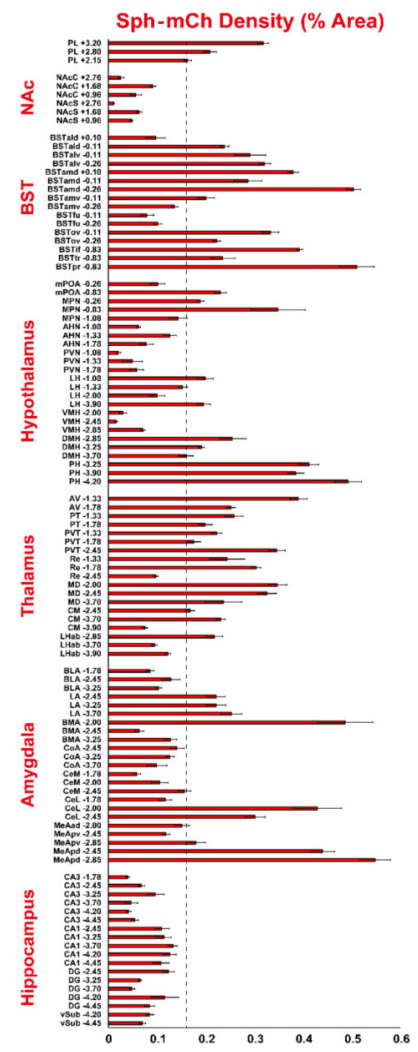

Figure 6.

Case 14 synaptophysin-mCherry density (percent area) throughout the limbic forebrain. Values expressed are mean \pm SEM from serial high-magnification images. Dashed line represents case median percent density. Distances listed are relative to bregma. See index for abbreviations. 

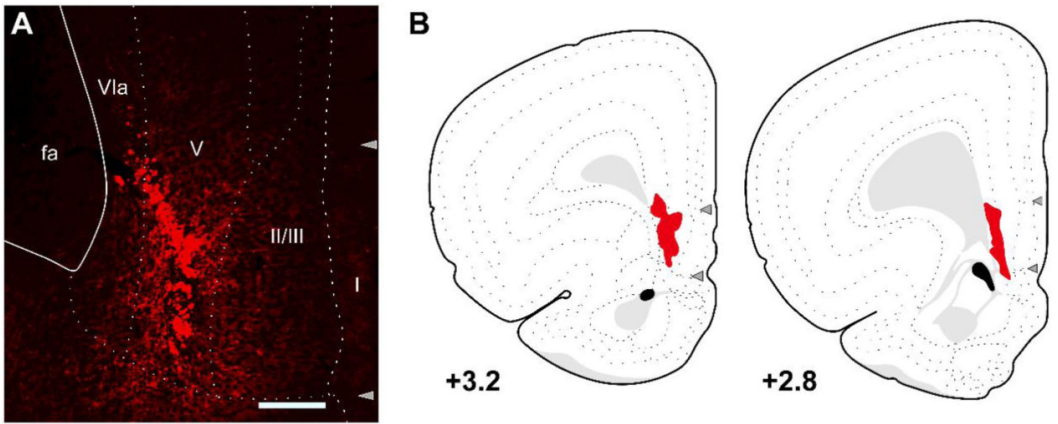

Figure 7.

Case 41 injection placement. Photomicrograph illustrates injection site for synaptophysinmCherry (red) in the right IL (A). The most rostral and caudal extents of the injections were mapped onto atlas templates (B; Swanson 2004). Distances listed are rostral to bregma (mm) and Roman numerals indicate cortical layers. Grey arrows indicate dorsal and ventral boundaries of IL. Scale bar: $200 \mu \mathrm{m}$. 


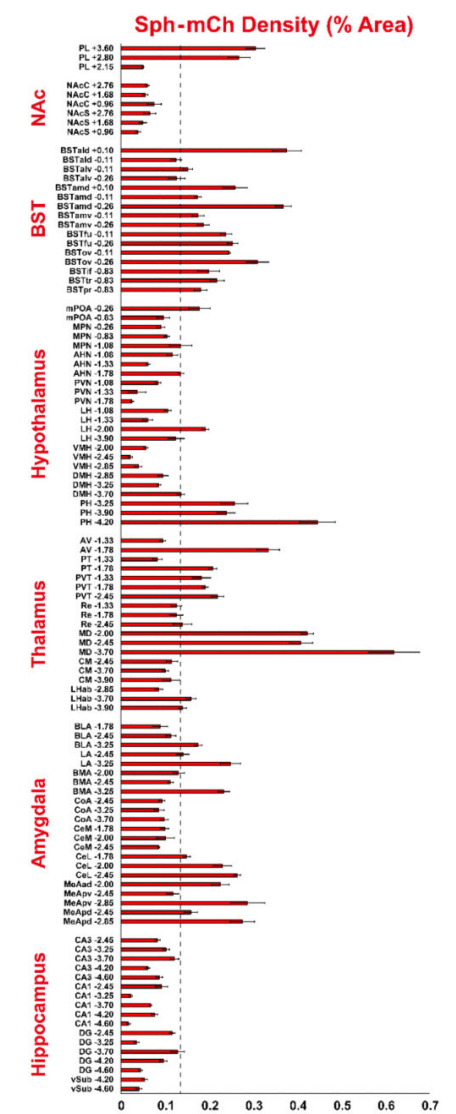

Figure 8.

Case 41 synaptophysin-mCherry density (percent area) in the limbic forebrain. Values expressed are mean \pm SEM from serial high-magnification images. Dashed line represents case median percent density. Distances listed are relative to bregma. See index for abbreviations. 


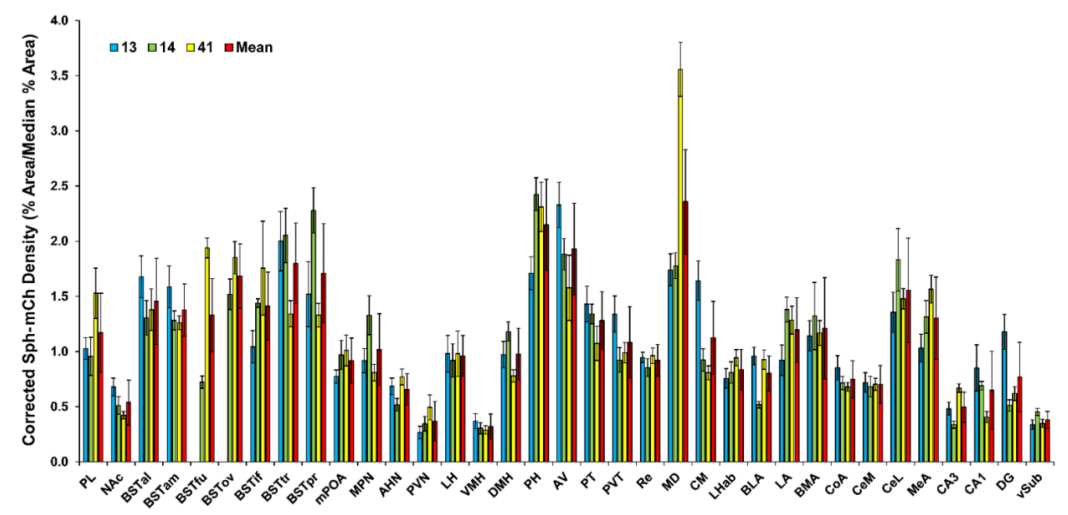

Figure 9.

Summary of structural projections. Median-corrected synaptophysin-mCherry densities are displayed for cases 13 (blue), 14 (green), and 41 (yellow) across the total rostral-caudal extension of each structure. The three cases were averaged to provide a mean (red) innervation density for each structure. Data are presented as mean \pm SEM. See index for abbreviations. 


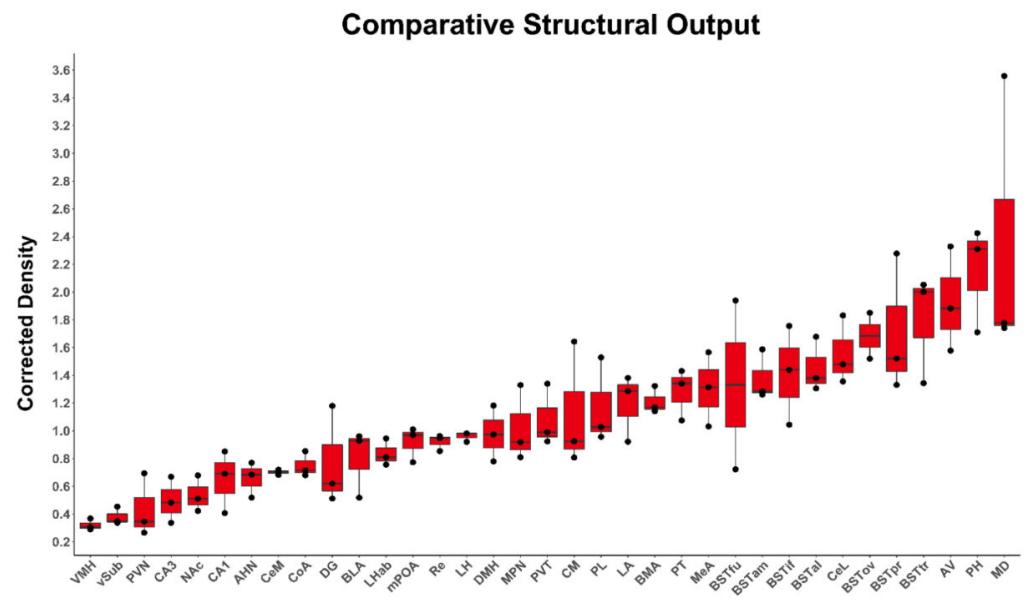

Figure 10.

Statistical comparison of structural output. The average corrected synaptophysin-mCherry density for each structure was mean-ranked with individual case values represented by black dots. See index for abbreviations. 


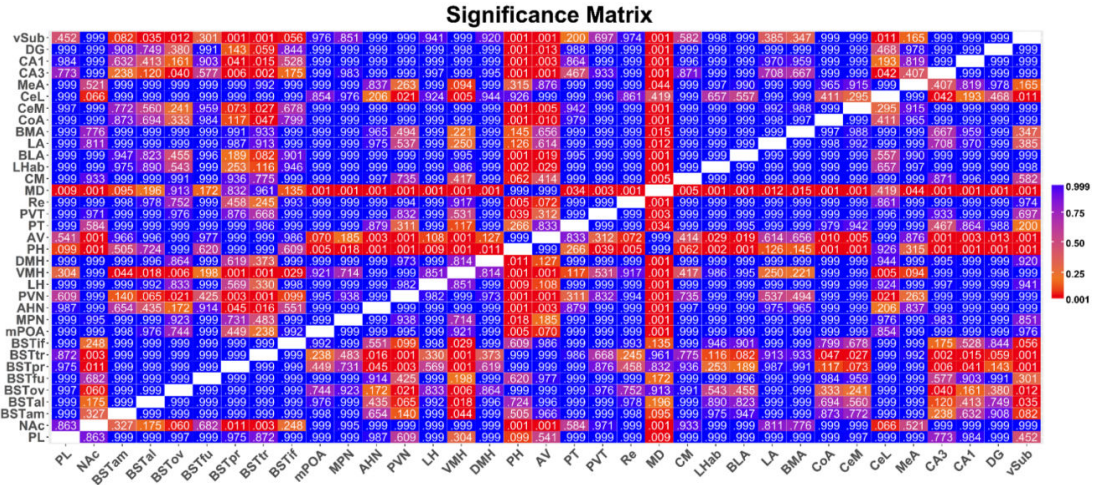

Figure 11.

Each structure was compared to all other structures, generating p-values to compose a significance matrix. Legend represents p-value range from 0.999 (blue) to 0.001 (red). All significant differences between structures are indicated by red matrix cells. See index for abbreviations. 

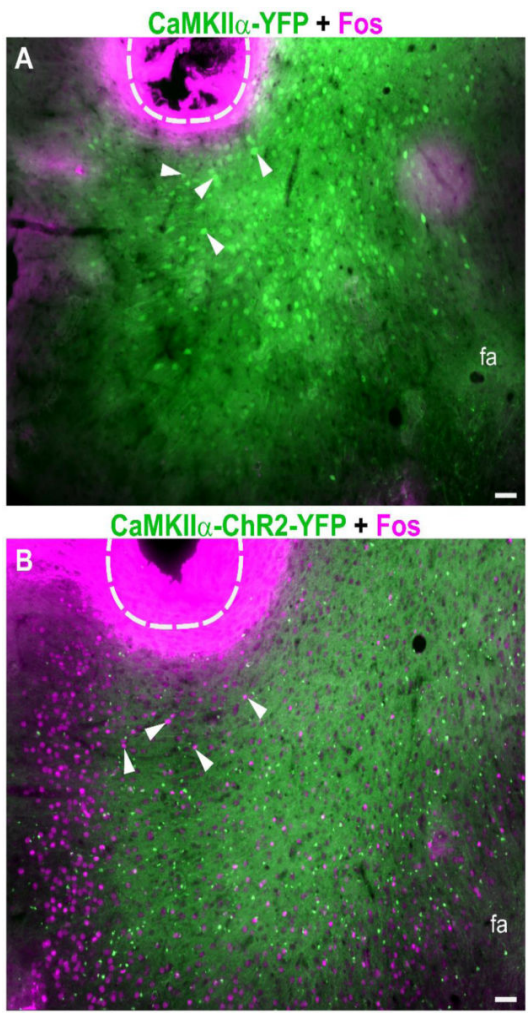

Figure 12.

Efficacy and selectivity of optogenetic parameters. AAVs coding for either CaMKIIa-YFP (A; green) or CaMKIIa-ChR2-YFP (B; green) were injected into IL. Following light stimulation, the immediate-early gene Fos (magenta) was expressed in ChR2-treated animals but not YFP-treated rats. Dashed white line indicates placement of optic fiber tip and white arrows point to cell bodies that express YFP (A) or Fos (B). CaMKIIa: $\mathrm{Ca}^{2+} /$ calmodulin- $^{2}$ dependent protein kinase II alpha; ChR2: channelrhodopsin-2; fa: corpus callosum anterior forceps; Scale bars: $100 \mu \mathrm{m}$. 

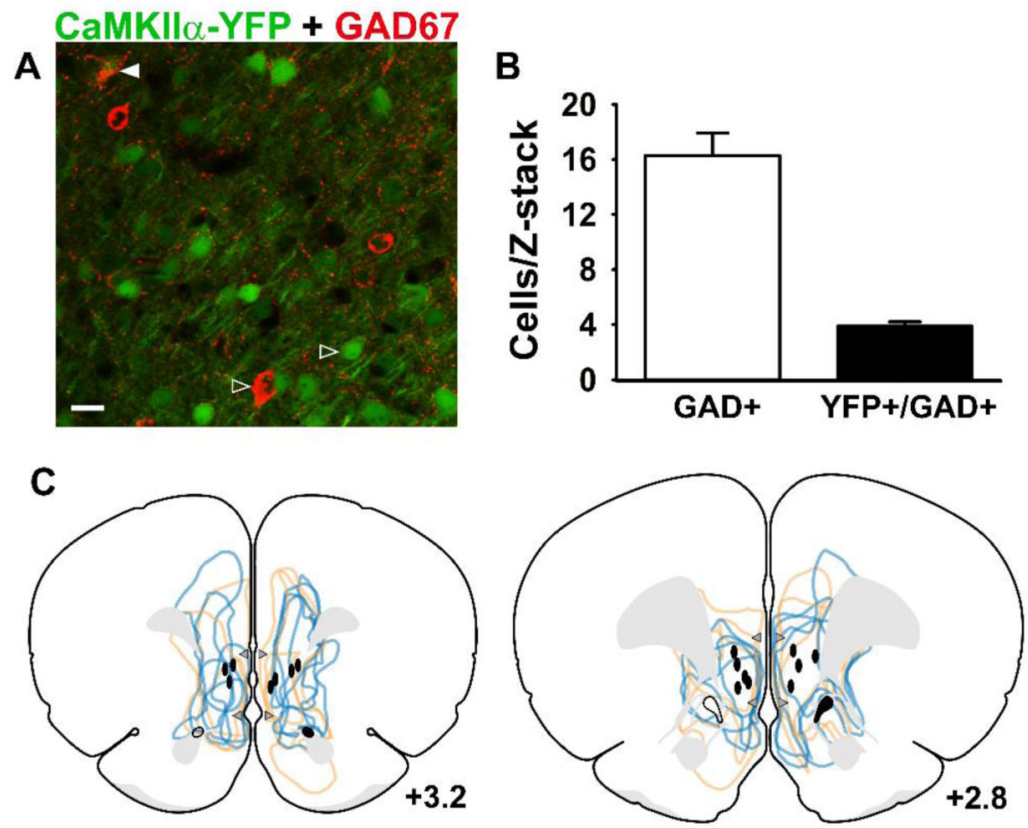

Figure 13.

Immunohistochemical labeling of the inhibitory neuron marker GAD67 (red) in CaMKIIaYFP-injected (green) tissue (A). Empty arrows indicate single labeling of either GAD or YFP and filled arrow indicates colabeling. Quantification indicated that CaMKIIa promotor expressed YFP in a small proportion of inhibitory neurons $(\mathbf{B})$. Placement of optic cannula fibers (grey ovals) were mapped onto atlas templates with individual viral injections mapped for YFP (yellow) and ChR2 (blue) groups (C). Distances listed are rostral to bregma (mm) and grey arrows indicate dorsal and ventral boundaries of IL. CaMKIIa: $\mathrm{Ca}^{2+} /$ calmodulindependent protein kinase II alpha; ChR2: channelrhodopsin-2; GAD67: glutamic acid decarboxylase, $67 \mathrm{kDa}$ isoform. Scale bar: $20 \mu \mathrm{m}$. 
Fra: YFP

A

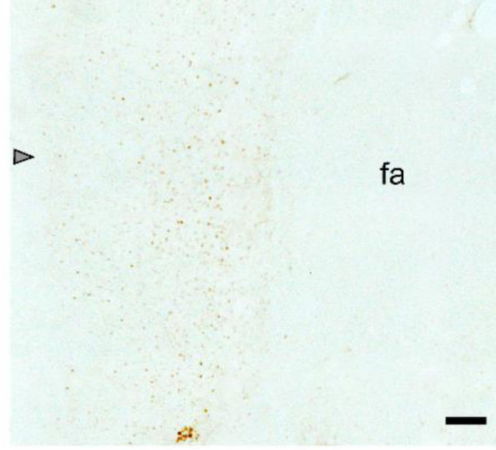

C

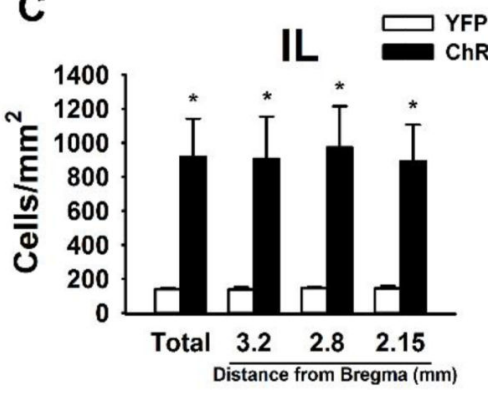

Fra: ChR2

B

fa

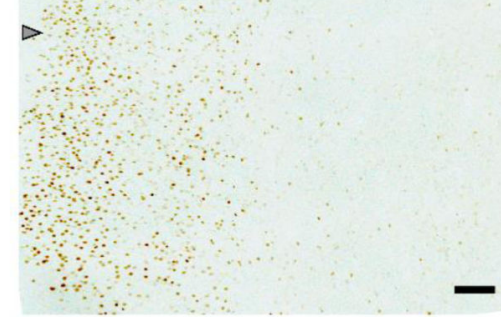

D

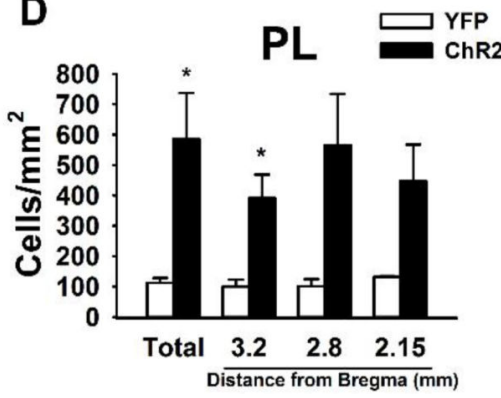

Figure 14.

Distribution of Fra-positive cells in the mPFC of rats injected with CaMKIIa-YFP $(\mathbf{A} ; \mathrm{n}=$ 3) or CaMKIIa-ChR2 (B; $n=5)$. Robust cellular activation is evident in ChR2 rats.

Quantification of Fra-positive cell density indicated a significant increase in the IL (C) and, to a lesser extent, the PL (D). Data are presented as mean \pm SEM. Grey arrows indicate dorsal boundary of the IL. fa: corpus callosum anterior forceps; Fra: fos-related antigen; IL: infralimbic cortex; PL: prelimbic cortex. Scale bars: $100 \mu \mathrm{m} .{ }^{*} \mathrm{p}<0.05$ 


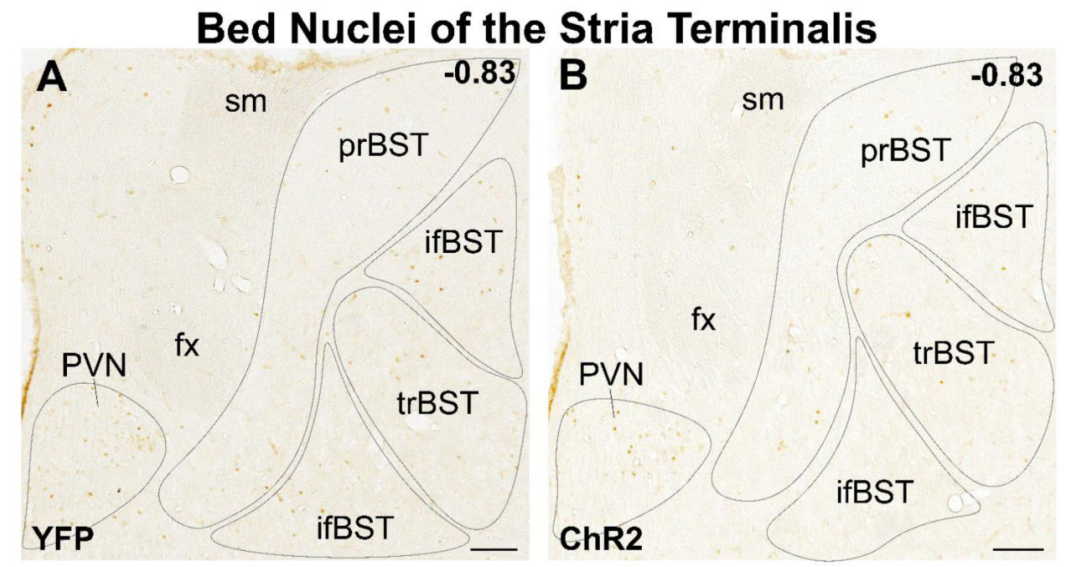

\section{Figure 15.}

Representative photomicrographs depicting Fra-immunoreactivity in the BST in rats expressing YFP (A) and ChR2 (B). There was no difference in Fra-positive cell density between YFP controls and ChR2. Distance listed is caudal to bregma (mm). ifBST:

interfascicular BST; prBST: principal BST; trBST: transverse BST; fx: fornix; PVN: paraventricular nucleus of the hypothalamus; sm: stria medullaris; Scale bars: $100 \mu \mathrm{m}$. 
A

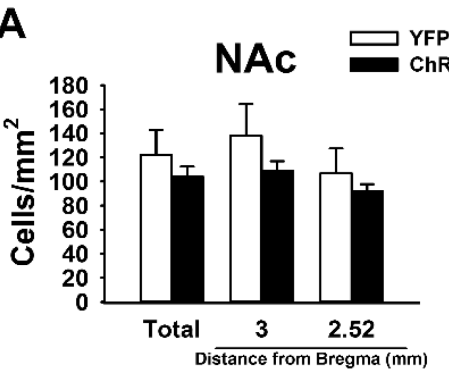

B

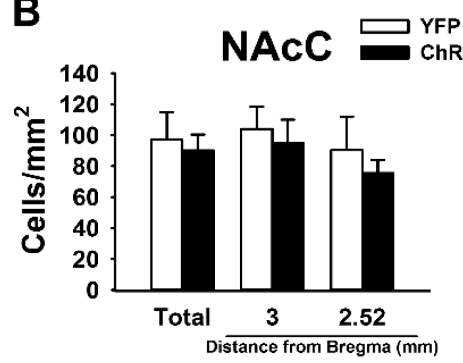

C

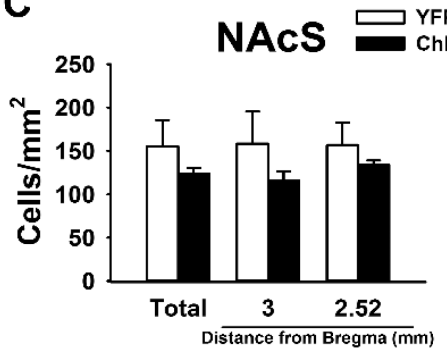

D

\section{E}
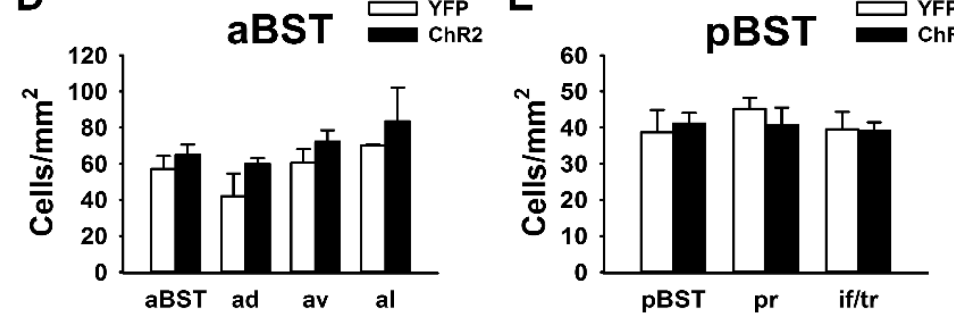

Figure 16.

There were no significant differences in Fra density between rats expressing YFP ( $\mathrm{n}=3)$ or ChR2 $(\mathrm{n}=5)$ in the NAc $(\mathbf{A}), \operatorname{NAcC}(\mathbf{B}), \operatorname{NAcS}(\mathbf{C}), \operatorname{aBST}(\mathbf{D})$, or pBST $(\mathbf{E})$. Data are presented as mean \pm SEM. aBST: anterior BST; ad: anterodorsal; al: anterolateral; av: anteroventral; if/tr: interfascicular/transverse; NAc: nucleus accumbens; NAcC: NAc core; NAcS: NAc shell; pBST: posterior BST; pr: principal. 
Hypothalamus

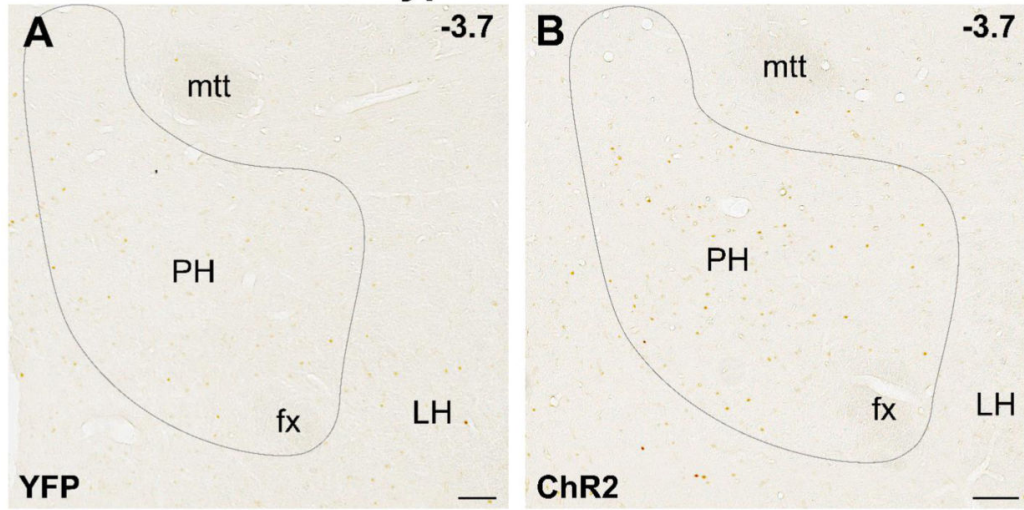

Figure 17.

Representative photomicrographs depicting Fra-immunoreactivity in the hypothalamus of rats expressing YFP (A) and ChR2 (B). Optogenetic activation of IL neurons increased Frapositive cell density in the PH. Distance listed is caudal to bregma (mm). fx: fornix; LH: lateral hypothalamus; mtt: mammillothalamic tract; $\mathrm{PH}$ : posterior hypothalamus; Scale bars: $100 \mu \mathrm{m}$. 

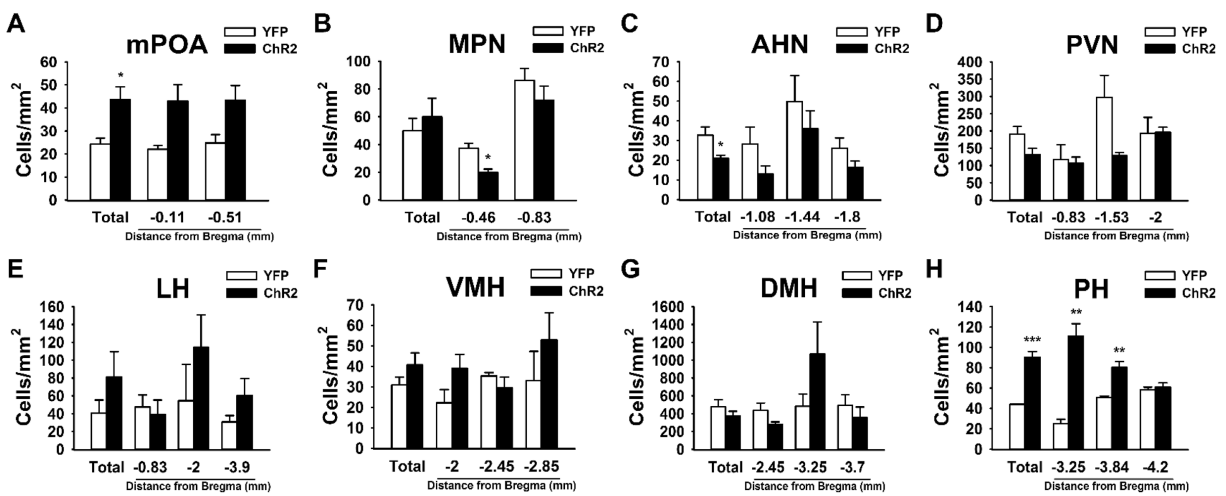

Figure 18.

Stimulation of IL in rats expressing ChR2 $(n=5)$ increased activation of mPOA (A) compared to YFP $(n=3)$. IL stimulation decreased activation of MPN $(\mathbf{B})$ and AHN $(\mathbf{C})$. There were no significant effects of optogenetic activation in IL on Fra immunoreactivity in the PVN (D), LH (E), VMH (F), or DMH (G). In contrast, IL activation increased Fra density in the PH $(\mathbf{H})$. Data are presented as mean \pm SEM. AHN: anterior hypothalamic nucleus; DMH: dorsomedial hypothalamus; LH: lateral hypothalamus; MPN: medial preoptic nucleus; mPOA: medial preoptic area; $\mathrm{PH}$ : posterior hypothalamic nucleus; PVN: paraventricular nucleus of the hypothalamus; VMH: ventromedial hypothalamus. ${ }^{*} \mathrm{p}<0.05$, $* * \mathrm{p}<0.01, * * * \mathrm{p}<0.001$ 


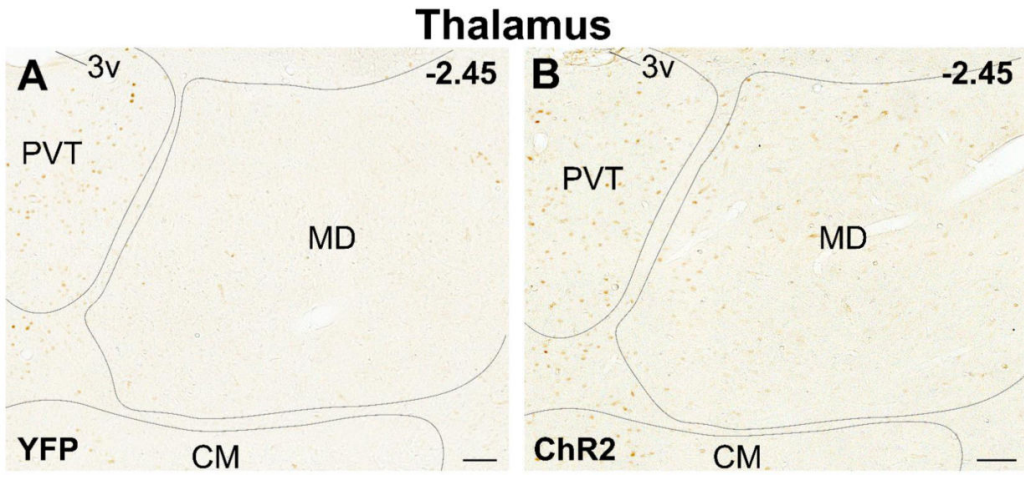

Figure 19.

Representative photomicrographs depicting Fra-immunoreactivity in the thalamus of rats expressing YFP (A) and ChR2 (B). Expression of ChR2 increased Fra-positive cell density in the PVT and MD compared to YFP. Distance listed is caudal to bregma (mm). 3v: third ventricle; CM: centromedial thalamus; MD: medialdorsal thalamus; PVT: paraventricular thalamus; Scale bars: $100 \mu \mathrm{m}$. 
A

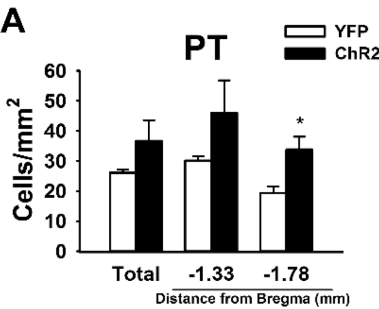

D

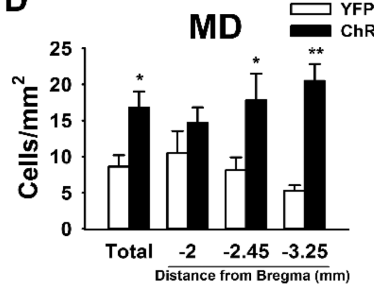

B

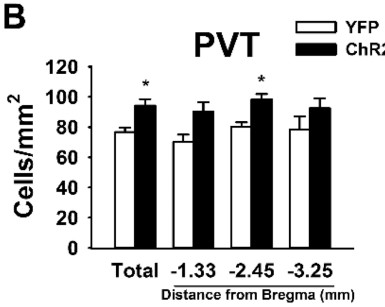

E

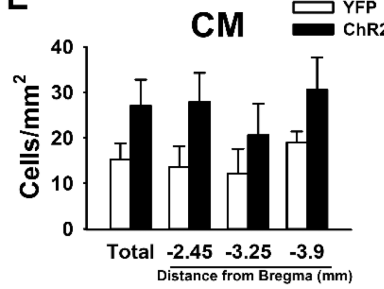

C

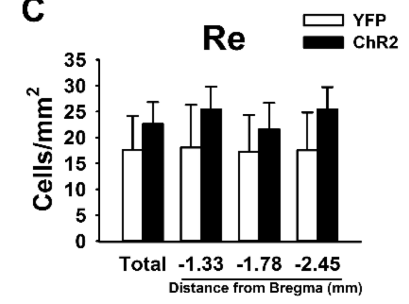

$F$

Figure 20.

Within the thalamus, IL stimulation increased activation in the PT (A) and PVT (B). There were no significant differences in the $\operatorname{Re}(\mathbf{C})$. The $M D(D)$ exhibited significant increases while the CM (E) and LHab (F) did not. Data are presented as mean \pm SEM. CM: centromedial thalamus; LHab: lateral habenula; MD: medialdorsal thalamus; PT: paratenial thalamus; PVT: paraventricular thalamus; Re: nucleus reunions. * $\mathrm{p}<0.05,{ }^{*} \mathrm{p}<0.01$ 


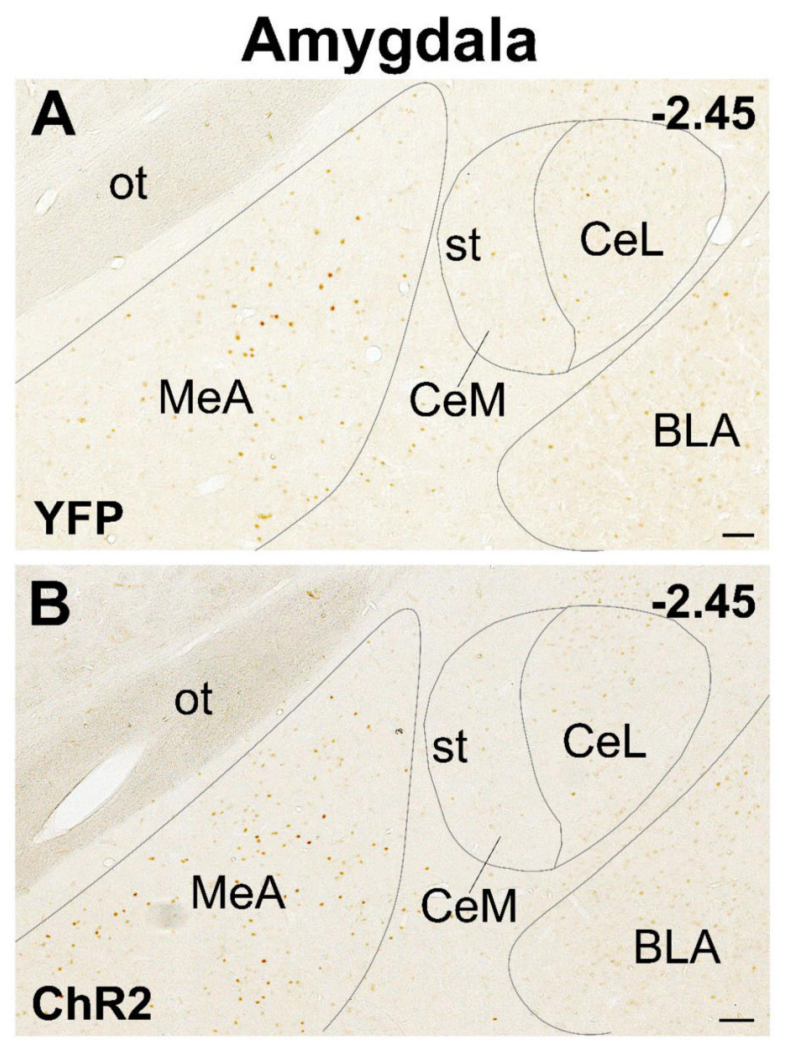

Figure 21.

Representative photomicrographs depicting Fra-immunoreactivity in the amygdala of rats expressing YFP (A) and ChR2 (B). ChR2-injected rats had elevated Fra-positive cell numbers in the CeA, primarily within the CeL. There was also an increase in Fra expression the MeA that was most prominent in the posterior portions of the nucleus. Distance listed is caudal to bregma (mm). BLA: basolateral amygdala; CeL: lateral subdivision of central amygdala; CeM: medial subdivision of central amygdala; MeA: medial amygdala; ot: optic tract; st: stria terminalis; Scale bars: $100 \mu \mathrm{m}$. 
A

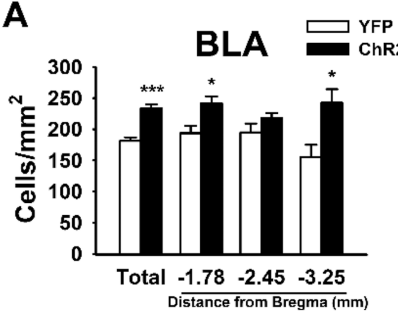

B

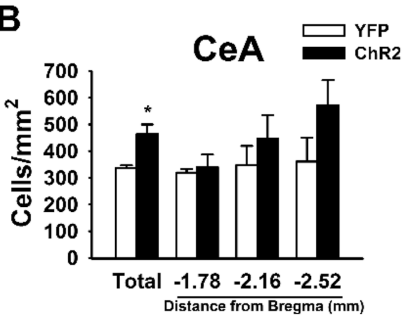

C

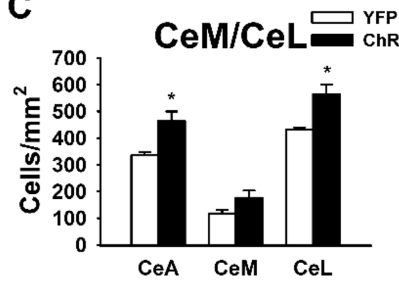

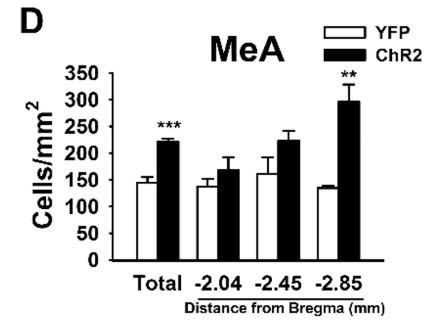

Figure 22.

Stimulation of the IL in rats expressing ChR2 $(\mathrm{n}=5)$ increased activation in the BLA (A) and CeA (B) compared to YFP $(n=3)$. The effects in the CeA were mediated by the CeL (C). IL activation also increased Fra expression in the MeA (D). Data are presented as mean \pm SEM. BLA: basolateral amygdala; CeA: central nucleus of the amygdala; $\mathrm{CeL}$ : lateral division of CeA; CeM: medial division of CeA; MeA: medial amygdala. * $\mathrm{p}<0.05,{ }^{* *} \mathrm{p}<$ $0.01, * * * \mathrm{p}<0.001$ 


\section{Hippocampus}

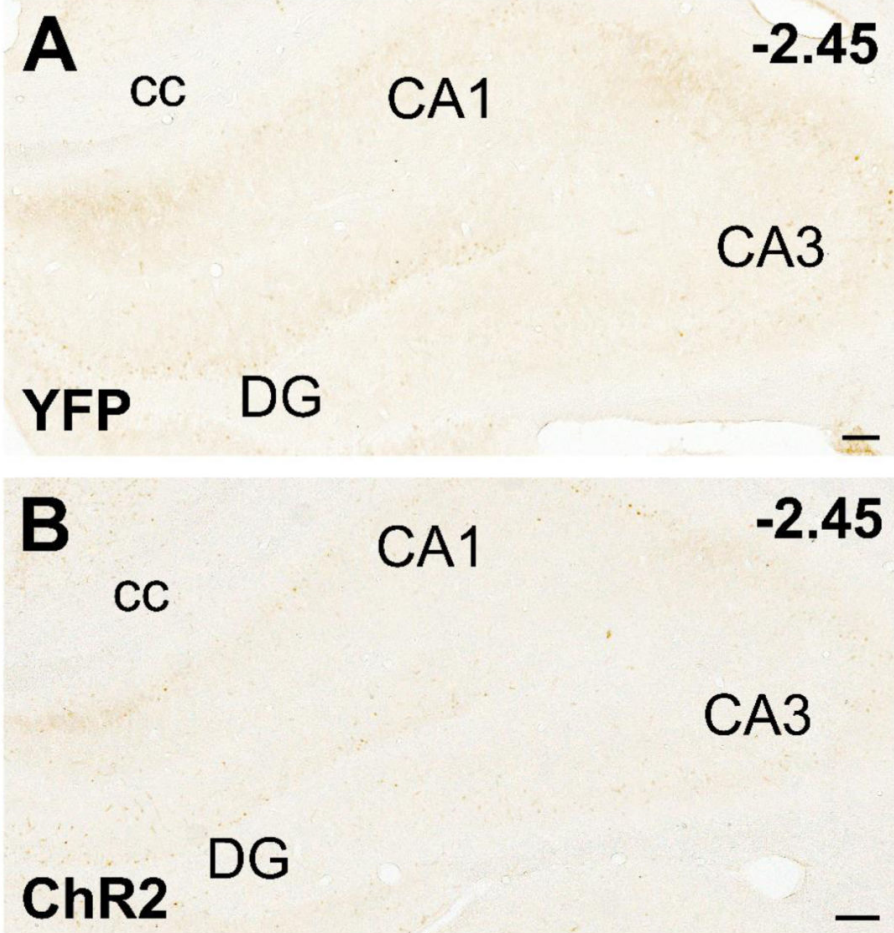

Figure 23.

Representative photomicrographs depicting Fra-immunoreactivity in the hippocampus of rats expressing YFP (A) and ChR2 (B). There was no difference in Fra-positive cell density between YFP controls and ChR2. Distance listed is caudal to bregma (mm). CA1: cornu ammonis field 1; CA3: cornu ammonis field 3; cc: corpus callosum; DG: dentate gyrus; Scale bars: $100 \mu \mathrm{m}$. 

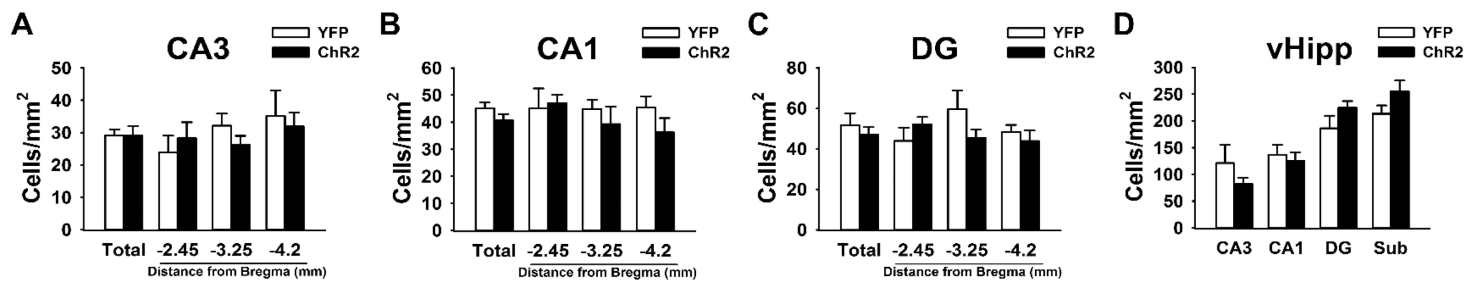

Figure 24.

There were no significant differences in Fra density between rats expressing YFP $(\mathrm{n}=3)$ or ChR2 $(\mathrm{n}=5)$ in the CA3 (A), CA1 (B), DG (C), or vHipp (D). Data are presented as mean \pm SEM. vHipp: ventral hippocampus. 


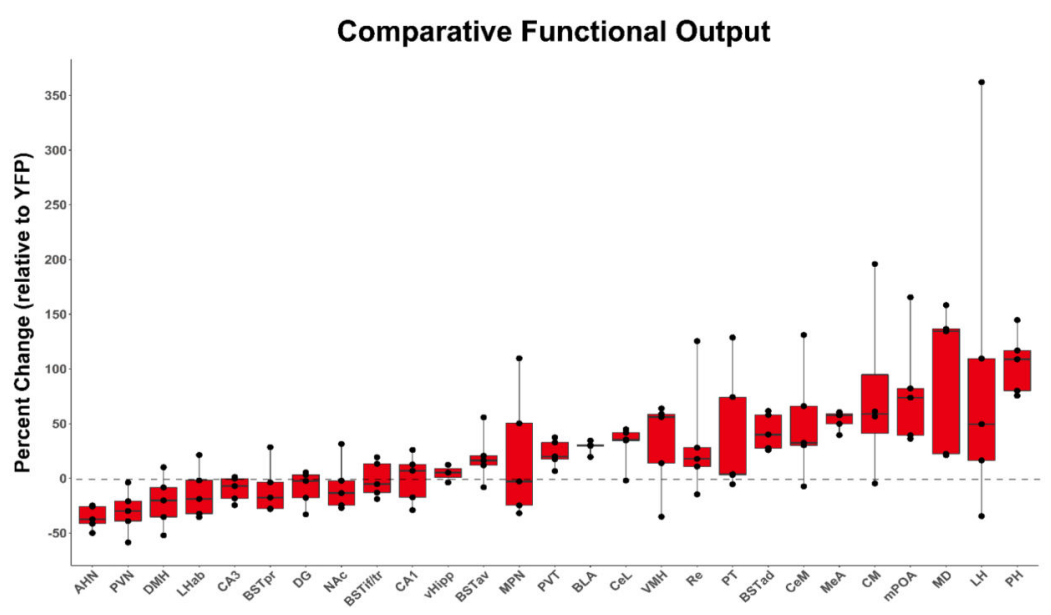

Figure 25.

Statistical comparison of functional output. The average percent change in Fra density between ChR2 and YFP was mean-ranked for each structure, with individual case values represented by black dots. See index for abbreviations. 


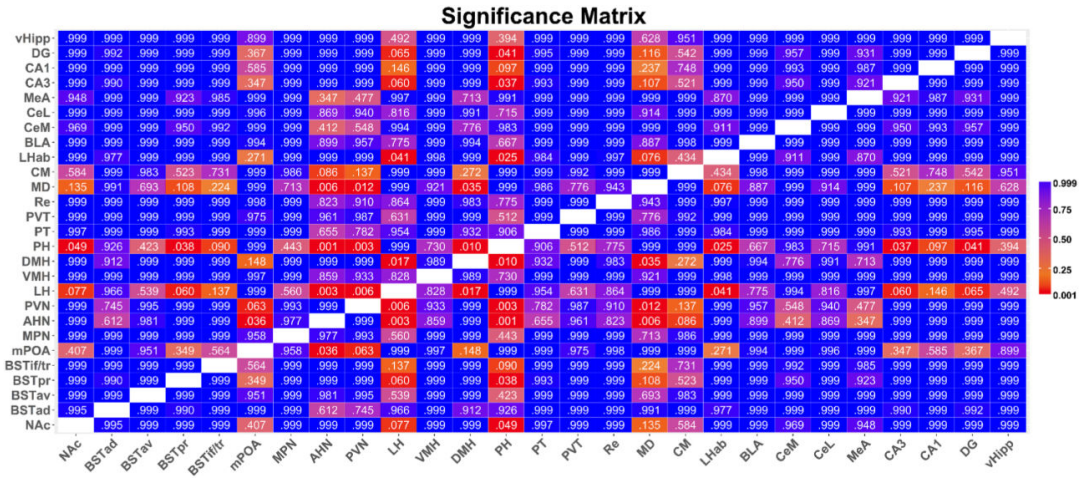

Figure 26.

Each structure was compared to all other structures, generating p-values to compose a significance matrix. Legend represents p-value range from 0.999 (blue) to 0.001 (red). All significant differences between structures are indicated by red matrix cells. See index for abbreviations. 
Table 1.

Summary of regions with significant difference between YFP and ChR2 in Fra immunoreactivity. ( $\uparrow$ ) or $(\downarrow)$ indicate significant increase or decrease, $(\leftrightarrow)$ no change, and (--) not assessed. See index for abbreviations.

\begin{tabular}{|c|c|c|c|c|}
\hline Structure & Total & Rostral & Middle & Caudal \\
\hline \multicolumn{5}{|l|}{$\mathrm{PFC}$} \\
\hline IL & $\uparrow$ & $\uparrow$ & $\uparrow$ & $\uparrow$ \\
\hline PL & $\uparrow$ & $\uparrow$ & $\leftrightarrow$ & $\leftrightarrow$ \\
\hline \multicolumn{5}{|c|}{ Accumbens } \\
\hline NAc & $\leftrightarrow$ & $\leftrightarrow$ & -- & $\leftrightarrow$ \\
\hline Core & $\leftrightarrow$ & $\leftrightarrow$ & -- & $\leftrightarrow$ \\
\hline Shell & $\leftrightarrow$ & $\leftrightarrow$ & -- & $\leftrightarrow$ \\
\hline \multicolumn{5}{|c|}{ Bed Nucleus } \\
\hline aBST & $\leftrightarrow$ & -- & -- & -- \\
\hline $\mathrm{ad}$ & $\leftrightarrow$ & -- & -- & -- \\
\hline av & $\leftrightarrow$ & -- & -- & -- \\
\hline al & $\leftrightarrow$ & -- & -- & -- \\
\hline pBST & $\leftrightarrow$ & -- & -- & -- \\
\hline $\mathrm{pr}$ & $\leftrightarrow$ & -- & -- & -- \\
\hline if $/ \mathrm{tr}$ & $\leftrightarrow$ & -- & -- & -- \\
\hline \multicolumn{5}{|c|}{ Hypothalamus } \\
\hline $\mathrm{mPOA}$ & $\uparrow$ & $\leftrightarrow$ & -- & $\leftrightarrow$ \\
\hline MPN & $\leftrightarrow$ & $\downarrow$ & -- & $\leftrightarrow$ \\
\hline AHN & $\downarrow$ & $\leftrightarrow$ & $\leftrightarrow$ & $\leftrightarrow$ \\
\hline PVN & $\leftrightarrow$ & $\leftrightarrow$ & $\leftrightarrow$ & $\leftrightarrow$ \\
\hline VMH & $\leftrightarrow$ & $\leftrightarrow$ & $\leftrightarrow$ & $\leftrightarrow$ \\
\hline LH & $\leftrightarrow$ & $\leftrightarrow$ & $\leftrightarrow$ & $\leftrightarrow$ \\
\hline $\mathrm{DMH}$ & $\leftrightarrow$ & $\leftrightarrow$ & $\leftrightarrow$ & $\leftrightarrow$ \\
\hline PH & $\uparrow$ & $\uparrow$ & $\uparrow$ & $\leftrightarrow$ \\
\hline \multicolumn{5}{|l|}{ Thalamus } \\
\hline PT & $\leftrightarrow$ & $\leftrightarrow$ & -- & $\uparrow$ \\
\hline PVT & $\uparrow$ & $\leftrightarrow$ & $\uparrow$ & $\leftrightarrow$ \\
\hline $\operatorname{Re}$ & $\leftrightarrow$ & $\leftrightarrow$ & $\leftrightarrow$ & $\leftrightarrow$ \\
\hline MD & $\uparrow$ & $\leftrightarrow$ & $\uparrow$ & $\uparrow$ \\
\hline $\mathrm{CM}$ & $\leftrightarrow$ & $\leftrightarrow$ & $\leftrightarrow$ & $\leftrightarrow$ \\
\hline LHab & $\leftrightarrow$ & $\leftrightarrow$ & $\leftrightarrow$ & $\leftrightarrow$ \\
\hline \multicolumn{5}{|l|}{ Amygdala } \\
\hline BLA & $\uparrow$ & $\uparrow$ & $\leftrightarrow$ & $\uparrow$ \\
\hline $\mathrm{CeA}$ & $\uparrow$ & $\leftrightarrow$ & $\leftrightarrow$ & $\leftrightarrow$ \\
\hline $\mathrm{CeM}$ & $\leftrightarrow$ & -- & -- & -- \\
\hline $\mathrm{CeL}$ & $\uparrow$ & -- & -- & -- \\
\hline $\mathrm{MeA}$ & $\uparrow$ & $\leftrightarrow$ & $\leftrightarrow$ & $\uparrow$ \\
\hline Dorsal Hip & & & & \\
\hline
\end{tabular}




\begin{tabular}{|c|c|c|c|c|c|}
\hline \multirow{4}{*}{$\frac{D}{\stackrel{D}{\underline{D}}}$} & Structure & Total & Rostral & Middle & Caudal \\
\hline & $\mathrm{CA} 3$ & $\leftrightarrow$ & $\leftrightarrow$ & $\leftrightarrow$ & $\leftrightarrow$ \\
\hline & CA1 & $\leftrightarrow$ & $\leftrightarrow$ & $\leftrightarrow$ & $\leftrightarrow$ \\
\hline & DG & $\leftrightarrow$ & $\leftrightarrow$ & $\leftrightarrow$ & $\leftrightarrow$ \\
\hline$\frac{7}{2}$ & Ventral Hipp & & & & \\
\hline गे & $\mathrm{CA} 3$ & $\leftrightarrow$ & -- & -- & -- \\
\hline$\frac{\vec{c}}{\infty}$ & CA1 & $\leftrightarrow$ & -- & -- & -- \\
\hline 을. & DG & $\leftrightarrow$ & -- & -- & -- \\
\hline ర్․ & Sub & $\leftrightarrow$ & -- & -- & -- \\
\hline
\end{tabular}

